\title{
MOVING BOUNDARY PROBLEMS
}

\author{
SUNČICA ČANIĆ
}

\begin{abstract}
Moving boundary problems are ubiquitous in nature, technology, and engineering. Examples include the human heart and heart valves interacting with blood flow, biodegradable microbeads swimming in water to clean up water pollution, a micro camera in the human intestine used for early colon cancer detection, and the design of next-generation vascular stents to prop open clogged arteries and to prevent heart attacks. These are time-dependent, dynamic processes, which involve the interaction between fluids and various structures. Analysis and numerical simulation of fluid-structure interaction (FSI) problems can provide insight into the "invisible" properties of flows and structures, and can be used to advance design of novel technologies and improve the understanding of many physical and biological phenomena. Mathematical analysis of FSI models is at the core of this understanding. In this paper we give a brief survey of recent progress in the area of mathematical well-posedness for moving boundary problems describing fluid-structure interaction between incompressible, viscous fluids and elastic, viscoelastic, and rigid solids.
\end{abstract}

\section{Contents}

1. Introduction

2. FSI with elastic structures

3. The arbitrary Lagrangian-Eulerian (ALE) mappings

4. Recent results and open problems in FSI with elastic structures

5. Compactness

6. FSI with rigid bodies

7. Recent results and open problems in FSI with rigid solids

8. Finite-time contact

About the author

References

\section{INTRODUCTION}

Moving boundary problems are time-dependent problems describing the motion of a quantity such as fluid in a domain that is moving due to, e.g., the domain's exterior boundary motion or the motion of an immersed structure within the fluid, or both. An immersed structure within the fluid defines an interior fluid domain

Received by the editors June 1, 2020.

2010 Mathematics Subject Classification. Primary 76D05, 76D03; Secondary 74F10, 76D27.

Key words and phrases. Moving boundary problems, fluid-structure interaction.

The author was supported in part by NSF Grants DMS-1853340 and DMS-1613757. 
boundary described by the fluid-structure interface location, which is, in general, time dependent. Moving boundary problems can be classified into two types: The first type is a moving boundary problem in which the domain boundary motion is given a priori. The second is a moving boundary problem in which the motion of the domain boundary is not known a priori, but this is one of the unknowns in the problem. In both cases the flow inside the domain is strongly affected by the motion of the domain boundary, while in the second case the motion of the domain boundary is simultaneously adjusted as it is being impacted by the flow inside the domain. In this case two sets of coupling conditions need to be prescribed to capture this two-way coupling: the kinematic condition describing the coupling of the kinematic quantities, such as velocity and the dynamic coupling condition describing the dynamic balance of forces. Depending on the problem at hand, this two-way coupling can be evaluated along the "current" location of the moving boundary, or along the fixed domain boundary. The coupling along a fixed domain boundary is said to be linear (i.e., it is linearized around the fixed, reference domain configuration), while the coupling along the current location of the moving boundary not known a priori is said to be nonlinear. See Figure 1. Needless to say, the nonlinear coupling between the flow and boundary motion gives rise to an exceedingly complicated nonlinear moving boundary problem, for which the theory of existence and uniqueness of solutions, and their continuous dependence on data, has only recently become the focus of a systematic mathematical study. Thus, it is of major interest to develop a general framework for the study of solutions to moving boundary problems.
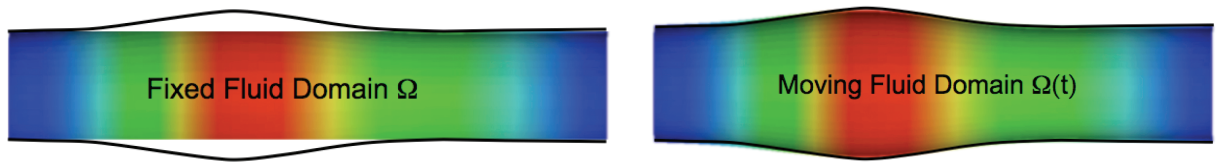

FIGURE 1. Fluid flow in a cylinder moving from left to right. The colors show the magnitude of pressure. The figure on the left shows the fluid domain in a linearly coupled fluid-structure interaction problem. The figure on the right shows the fluid domain in the nonlinearly coupled fluid-structure interaction problem.

In this paper we survey some recent developments and open problems in this area. In particular, we focus on problems arising from the interaction between incompressible, viscous fluids and elastic, viscoelastic, or rigid solids (also referred to as structures). Fluid-structure interaction (FSI) problems are ubiquitous in nature, technology, and engineering: from environmental science, where pollutant concentration is studied in aquifers through poroelastic media, to biomedical engineering and cardiovascular medicine, where, e.g., designs for vascular stents for treatment of coronary artery disease and heart valve replacement are studied.

Interestingly enough, even though the mathematical theory of the motion of bodies in a liquid is one of the oldest and most classical problems in fluid mechanics, mathematicians have only recently become interested in a systematic study of the basic problems related to fluid-structure interaction. One reason for this may be that problems of this type are notoriously difficult to study. In addition to the nonlinearity in the fluid and possibly the structure equations, the nonlinear coupling 
between the fluid and structure motion may give rise to strong geometric nonlinearities. Mathematical study of the existence of solutions to the coupled problems must account for nonlinearities due to the strong energy exchange between the fluid and (elastic) structure motion in problems with nonlinear coupling, and they must employ novel compactness arguments to deal with the nonlinearities. Due to the fluid domain motion, the compactness results must hold for a family of operators defined on time-dependent function spaces associated with moving domains not known a priori. Additionally, the compactness arguments must account for the fact that the coupled problem involves two sets of equations of different type (parabolic vs. hyperbolic) accounting for the different physics in the problem. Crucial for the existence proofs and the compactness arguments is to make use of the parabolic regularizing effects (by the fluid viscosity) to keep the high frequency oscillations of the (hyperbolic) structure under control.

In existence proofs, and in numerical schemes, an additional difficulty is imposed by the incompressibility of the fluid. The main difficulties in existence proofs relate to the construction of divergence-free extensions of fluid velocity to a larger domain containing all the moving domains and to obtaining quantitative estimates of the extensions in terms of the changing geometry. Incompressibility is intimately related to the pressure, and pressure is a major component of the load (i.e., contact force) exerted by the fluid onto the solid. Designing constructive existence proofs and numerical schemes that approximate the load "correctly" is a key ingredient for the stability of constructive solution schemes. In particular, the fluid surrounding the structure affects the structure motion as an extra mass that the structure must displace when moving within a fluid. This has long been known in engineering as the added mass effect. Not accounting for the added mass effect can have negative impact on the stability of partitioned FSI schemes, and this is a well-known problem within FSI problems for which the density of the structure is less than or equal to that of the fluid, i.e., for which the structure is light with respect to the fluid. The added mass is a leading order effect in biofluidic FSI problems, since biological tissues (structures) have density which is approximately the same as that of the surrounding fluid. A failure to account for this effect is associated with the lack of uniform energy estimates in constructive existence proofs for nonlinearly coupled FSI problems.

The question of global-in-time existence of solutions to moving boundary problems is affected by two open problems. One is inherited from the NavierStokes equations and the open outstanding question of global existence of strong solutions. The other is related to the so-called no-collision paradox: global weak solution existence results for moving boundary problems are typically obtained until a possible fluid domain degeneracy occurs, such as, e.g., collapse of the cylindrical tube leading to the cross-sectional area of the tube approaching zero. The problems of finite-time contact between elastic bodies in a viscous, incompressible fluid remains an open question - the no-collision paradox. As we shall see below, various questions related to the no-collision paradox are being investigated, including the possibility of finite time contact for classical models, investigation and design of mathematical models that would allow finite time contact, and the type of boundary conditions (no-slip versus slip) for which finite-time contact may occur.

Nonlinearities in the coupled FSI problem also affect the study of uniqueness of solutions. It is not surprising that uniqueness of weak solutions to the coupled 
FSI problems is still largely an outstanding open problem, since even in the case of classical three-dimensional Navier-Stokes equations, the uniqueness of the LerayHopf weak solutions has not been resolved. However, recent advances in this area are significant, and we summarize those results below.

To explain the main challenges in more detail, we present a benchmark problem for FSI involving elastic structures as well as a benchmark problem for FSI involving rigid solids, and we provide a literature review of the recent results.

\section{FSI With ElAStiC STRUCTURES}

Although the development of numerical methods for fluid-structure interaction problems started almost 40 years ago (see, e.g., [8, 9, 41, 42, 46, 52, 73, 76, 78, 86, 87, 90, 113, 114, 117, 131 and the references therein), the development of existence theory for FSI problems started less than 20 years ago. We state a benchmark problem in this field and summarize some recent results and open problems.

Description of the main problem. To describe the interaction between a fluid and an elastic (or viscoelastic) structure across a moving interface mathematically, two types of coupling conditions have to be prescribed. This contrasts with classical fluid dynamics problems defined on fixed domains, where only one boundary condition (e.g., the no-slip condition) is sufficient to define the problem. As mentioned earlier, the two sets of coupling conditions describe the following: (1) how the kinematic quantities, such as velocity, are coupled (the kinematic coupling condition, e.g., no-slip); and (2) the elastodynamics of the fluid-structure interface (the dynamic coupling condition). While the precise form of the kinematic and dynamic coupling conditions depends on the particular application at hand, the most common coupling is done via the no-slip kinematic condition (which states that the fluid and structure velocities are continuous across the moving interface) and the dynamic coupling condition (which states that the fluid-structure interface, namely the moving boundary, is driven by the jump in traction, i.e., normal stress, across the interface). For problems in which one expects small interface displacements and small displacement gradients, the coupling conditions may be evaluated at a fixed interface without changing the fluid domain; namely, the fluid and structure may be linearly coupled [10,11,45, 93. For problems where this may not be a good approximation of reality, the coupling conditions must be evaluated across the moving interface, giving rise to an additional nonlinearity in the problem, which is due to the change of geometry of the moving boundary; namely, the fluid and structure are nonlinearly coupled. In the latter case the fluid domain is a function of time, and additionally, it is not known a priori since it depends on the unknowns in the problem, namely, the displacement of the fluid-structure interface.

The geometric nonlinearity, associated with the fluid domain motion not known a priori, presents one of the major, new difficulties in studying this class of problems mathematically.

2.1. FSI benchmark problem with no-slip. The simplest example of a moving boundary problem with nonlinear coupling involving a deformable (elastic) structure is a benchmark problem derived from modeling blood flow in a segment of an artery. The fluid domain is a cylinder with an elastic (viscoelastic) lateral boundary. For simplicity, we present the problem in two dimensions, although three-dimensional versions of the problem have been studied in, e.g., 103. In this 
benchmark problem, we will be assuming that the lateral boundary is thin with small thickness, $h \ll 1$, and with the reference configuration $\Gamma$ corresponding to a straight cylinder of length $L$ and radius $R$ :

$$
\Gamma=\{(z, R) \mid z \in(0, L)\} .
$$

In most literature involving FSI with thin structures (except for the recent results in [23, 106]), the lateral boundary is assumed to displace only in the vertical (normal, transverse) direction, rendering longitudinal displacement negligible. By using $\eta$ to denote the vertical component of displacement, the fluid domain $\Omega^{\eta}(t)$ can be described by

$$
\Omega^{\eta}(t)=\left\{(z, r) \in \mathbb{R}^{2} \mid z \in(0, L), r \in(0, R+\eta(t, z)\},\right.
$$

where $R$ is the radius of the reference cylinder. The reference fluid domain will be denoted by $\Omega=(0, L) \times(0, R)$.

The fluid flow is modeled by the Navier-Stokes equations for an incompressible, viscous fluid, defined on a moving domain $\Omega^{\eta}(t)$, which are not known a priori,

$$
\left.\begin{array}{rl}
\rho_{f}\left(\partial_{t} \mathbf{u}+(\mathbf{u} \cdot \nabla) \mathbf{u}\right) & =\nabla \cdot \boldsymbol{\sigma} \\
\nabla \cdot \mathbf{u} & =0
\end{array}\right\} \text { in } \Omega^{\eta}(t), t \in(0, T)
$$

where $\boldsymbol{\sigma}$ is the Cauchy stress tensor, $\rho_{f}$ is the fluid density, and $\mathbf{u}=\mathbf{u}(\boldsymbol{x}, t)=$ $\left(u_{z}, u_{r}\right)$ is the fluid velocity. For Newtonian fluids

$$
\boldsymbol{\sigma}=-p \mathbf{I}+2 \mu \mathbf{D}(\mathbf{u})
$$

where $\mu$ is the dynamic viscosity coefficient and $\mathbf{D}(\mathbf{u})=\frac{1}{2}\left(\nabla \mathbf{u}+\nabla^{\tau} \mathbf{u}\right)$ is the symmetrized gradient of $\mathbf{u}$.

In this benchmark problem the flow is driven by the inlet and outlet dynamic pressure data, and the flow is normal to the inlet and outlet boundary $\Gamma_{\text {in }}=$ $\{0\} \times(0, R)$ and $\Gamma_{\text {out }}=\{L\} \times(0, R)$, respectively,

$$
\left.\begin{array}{rl}
p+\frac{\rho_{f}}{2}|u|^{2} & =P_{\text {in/out }}(t) \\
u_{r} & =0
\end{array}\right\} \quad \text { on } \Gamma_{\text {in/out }},
$$

where $P_{\text {in } / \text { out }} \in L_{\text {loc }}^{2}(0, \infty)$ are given. At the bottom boundary $\Gamma_{b}=(0, L) \times\{0\}$, the symmetry boundary conditions are prescribed,

$$
u_{r}=\partial_{r} u_{z}=0, \quad \text { on } \Gamma_{b} .
$$

See Figure 2, left.

Different inlet/outlet boundary conditions have been used in numerical simulations, including in Dirichlet data given in terms of the prescribed fluid velocity and in Neumann data given in terms of the prescribed normal stress. Prescribing the correct boundary conditions, especially at the outflow boundary, is very important in correctly capturing the physiological flow conditions within a subregion of the cardiovascular system that is being modeled [117. From the analysis point of view, it is now known that some of the numerically convenient outlet boundary conditions, such as the Neumann, or do nothing, outlet boundary conditions, may produce instabilities 132 or ruin well-posedness by producing multiple solutions to the steady flow, as was recently shown in [77. In this paper we consider dynamic pressure data (2.2) both at the inlet and at the outlet, which is a boundary condition that is consistent with the energy of the coupled problem. 

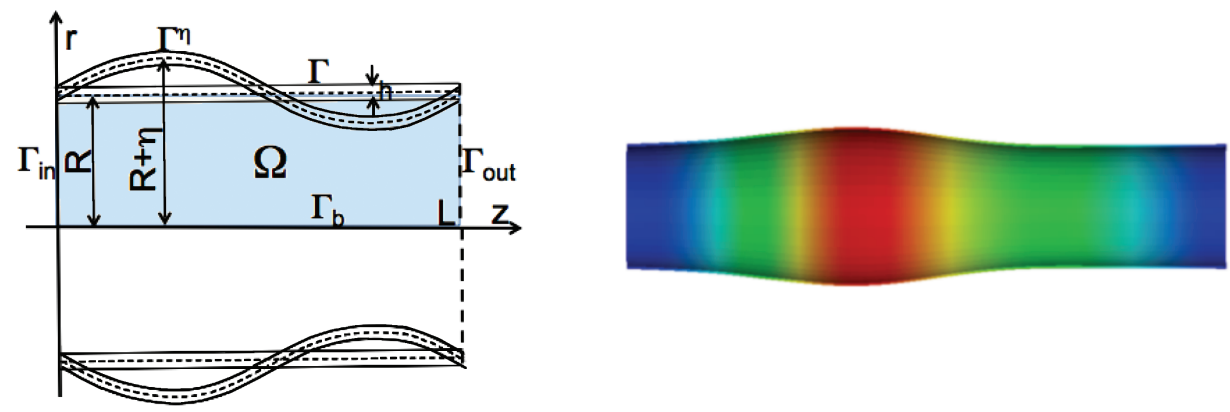

Figure 2. Left: A sketch of the fluid domain and the moving lateral boundary. Right: Pressure wave propagation in an elastic tube.

Under fluid loading, and possibly some external loading, the elastic cylinder deforms. See Figure 2, We denote by

$$
\Gamma^{\boldsymbol{\eta}}(t)=\{(t, z, R+\eta(t, z)) \mid z \in(0, L)\}
$$

the location of the deformed cylinder lateral boundary at time $t$. The elastic properties of the cylinder's lateral wall can be described by an operator $\mathcal{L}_{e}$, so that the elastodynamics problem, in Lagrangian formulation, can be written as

$$
\rho_{s} h \partial_{t t} \eta+\mathcal{L}_{e} \eta=f, \quad \text { on } \Gamma, t \in(0, T),
$$

where $\rho_{s}$ is the structure density, $h$ is the thin structure thickness, and $f$ is the vertical component of the external loading (force density) experienced by the elastic structure. The loading $f$ in the coupled problem will come from the jump in the normal stress across the structure, i.e., from the fluid load experienced by the structure (assuming that the external loading is zero). The operator $\mathcal{L}_{e}$ is associated with the elastic energy of the structure, such as the membrane or shell energy (see [102]), and it is typically continuous, positive-definite, and coercive on some Hilbert space $\chi$.

The coupling. The fluid flow influences the motion of the structure through traction forces (i.e., by the normal stress exerted onto the structure at $\Gamma^{\boldsymbol{\eta}}(t)$ ) while the structure influences the fluid through its inertial and elastic forces due to the structure motion and stretching/recoil. Additionally, the fluid and structure "feel" each other through the continuity of the fluid and structure velocities at the interface. Thus, the kinematic and dynamic coupling conditions are, respectively,

$$
\begin{array}{r}
\left(\partial_{t} \eta(t, z), 0\right)=\boldsymbol{u}(t, z, R+\eta(t, z)), \\
\rho_{s} h \partial_{t}^{2} \eta+\mathcal{L}_{e} \eta=-\left.J(\boldsymbol{\sigma n})\right|_{(t, z, R+\eta(t, z))} \cdot \mathbf{e}_{r},
\end{array}
$$

where $J=\sqrt{1+\left(\frac{\partial \eta}{\partial t}\right)^{2}}$, is the Jacobian of the transformation from Eulerian coordinates to Lagrangian coordinates, and $\mathbf{e}_{r}$ is the unit vector in the vertical direction. Here, we have assumed that external forcing onto the structure is zero. Generalizations to include external forcing due to the presence of another elastic structure or other types of forcing can be found in [104].

The geometric nonlinearity due to the fluid domain motion, described by the composite function $\boldsymbol{u}(t, z, R+\eta(t, z))$, is generally handled by introducing a family of 
mappings, parameterized by time, called the arbitrary Lagrangian-Eulerian (ALE) mappings, discussed in section 3 . In terms of ALE mappings, the trace of the fluid velocity on $\Gamma^{\eta}(t)$ is described by a composite function between the velocity and the ALE mapping.

Equations (2.1)-(2.7) define a nonlinear moving-boundary problem for the unknown functions $\mathbf{u}$ and $\eta$. The problem is supplemented with the initial conditions

$$
\mathbf{u}(0, .)=\mathbf{u}_{0}, \quad \eta(0, .)=\eta_{0}, \quad \partial_{t} \eta(0, .)=v_{0},
$$

satisfying the compatibility conditions

$$
\begin{gathered}
\mathbf{u}_{0}\left(z, R+\eta_{0}(z)\right)=v_{0}(z) \mathbf{e}_{r}, \quad z \in(\Gamma), \\
\eta_{0}(0)=\eta_{0}(L)=v_{0}(0)=v_{0}(L)=0, \\
R+\eta_{0}(z)>0, \quad z \in[0, L] .
\end{gathered}
$$

Thus, the benchmark nonlinear moving-boundary problem, which exemplifies the main difficulties associated with studying moving boundary problems with nonlinear coupling, can be summarized as follows. Find $\mathbf{u}=\left(u_{z}(t, z, r), u_{r}(t, z, r)\right)$, $p$, and $\eta(t, z)$ such that

$$
\begin{aligned}
& \left.\begin{array}{rl}
\rho_{f}\left(\partial_{t} \mathbf{u}+(\mathbf{u} \cdot \nabla) \mathbf{u}\right) & =\nabla \cdot \boldsymbol{\sigma}(\mathbf{u}, p) \\
\nabla \cdot \mathbf{u} & =0
\end{array}\right\} \text { in } \Omega^{\eta}(t), t \in(0, T), \\
& \left.\begin{array}{rl}
\left.\mathbf{u}\right|_{\Gamma^{\eta}(t)} & =\partial_{t} \eta \mathbf{e}_{r} \\
\rho_{s} h \partial_{t}^{2} \eta+\mathcal{L}_{e} \eta & =-\left.J^{\eta} \boldsymbol{\sigma} \mathbf{n}\right|_{\Gamma^{\eta}(t)} \cdot \mathbf{e}_{r}
\end{array}\right\} \text { on }(0, T) \times \Gamma, \\
& \left.\begin{array}{rl}
u_{r} & =0 \\
\partial_{r} u_{z} & =0
\end{array}\right\} \text { on }(0, T) \times \Gamma_{b}, \\
& \left.\begin{array}{rl}
p+\frac{\rho_{f}}{2}|u|^{2} & =P_{\text {in/out }}(t) \\
u_{r} & =0
\end{array}\right\} \text { on }(0, T) \times \Gamma_{\text {in } / \text { out }}, \\
& \eta(t, 0)=\partial_{z} \eta(t, 0)=\eta(t, L)=\partial_{z} \eta(t, L)=0 \quad \text { on }(0, T), \\
& \left.\begin{array}{rl}
\mathbf{u}(0, .) & =\mathbf{u}_{0} \\
\eta(0, .) & =\eta_{0} \\
\partial_{t} \eta(0, .) & =v_{0}
\end{array}\right\} \text { at } t=0 .
\end{aligned}
$$

The energy. This benchmark problem satisfies the formal energy inequality

$$
\frac{d}{d t} E(t)+D(t) \leq C\left(P_{\text {in }}(t), P_{\text {out }}(t)\right)
$$

where $E(t)$ denotes the sum of the kinetic energy of the fluid and of the structure, and the elastic energy of the membrane shell,

$$
E(t)=\frac{\rho_{f}}{2}\|\mathbf{u}\|_{L^{2}\left(\Omega^{\eta}(t)\right)}^{2}+\frac{\rho_{s} h}{2}\left\|\partial_{t} \eta\right\|_{L^{2}(\Gamma)}^{2}+\left\langle\mathcal{L}_{e} \eta, \eta\right\rangle,
$$

where $\left\langle\mathcal{L}_{e} \eta, \eta\right\rangle$ corresponds to the elastic energy of the structure, which for the cylindrical Koiter shell allowing only radial displacement reads

$$
\left\langle\mathcal{L}_{e} \eta, \eta\right\rangle:=\frac{1}{2}\left(C_{0}\|\eta\|_{L^{2}(\Gamma)}^{2}+C_{1}\left\|\partial_{z} \eta\right\|_{L^{2}(\Gamma)}^{2}+C_{2}\left\|\partial_{z}^{2} \eta\right\|_{L^{2}(\Gamma)}^{2}\right) .
$$

The term $D(t)$ captures dissipation due to fluid viscosity,

$$
D(t)=\mu\|\mathbf{D}(\mathbf{u})\|_{\left.L^{2}\left(\Omega^{\eta}(t)\right)\right)}^{2},
$$

and $C\left(P_{\text {in }}(t), P_{\text {out }}(t)\right)$ is a constant which depends only on the inlet and outlet pressure data, which are both functions of time. 
2.2. FSI benchmark problem with Navier-slip. While the assumption on the continuity of normal velocity components is reasonable for impermeable boundaries,

$$
\left(\partial_{t} \boldsymbol{\eta}-\boldsymbol{u}\right) \cdot \mathbf{n}=0 \quad \text { (nonpenetration condition), }
$$

the continuity of the tangential velocity component in the no-slip condition is justified only when molecular viscosity is considered [99]. Navier contested the no-slip condition for Newtonian fluids [109] when he claimed that the tangential, slip velocity should be proportional to the shear stress. For moving boundary problems this means that the jump in the tangential components of the fluid and solid velocities at the moving boundary is proportional to the shear stress,

$$
\left(\partial_{t} \boldsymbol{\eta}-\boldsymbol{u}\right) \cdot \boldsymbol{\tau}=\alpha \boldsymbol{\sigma} \mathbf{n} \cdot \boldsymbol{\tau} \quad \text { (Navier slip), }
$$

where $\mathbf{n}$ and $\boldsymbol{\tau}$ to denote the unit normal and tangent to the fluid domain boundary, respectively, $\boldsymbol{\sigma}$ is the fluid Cauchy stress tensor, and $\alpha$ is the proportionality constant known as the slip length; $1 / \alpha$ has the units of friction.
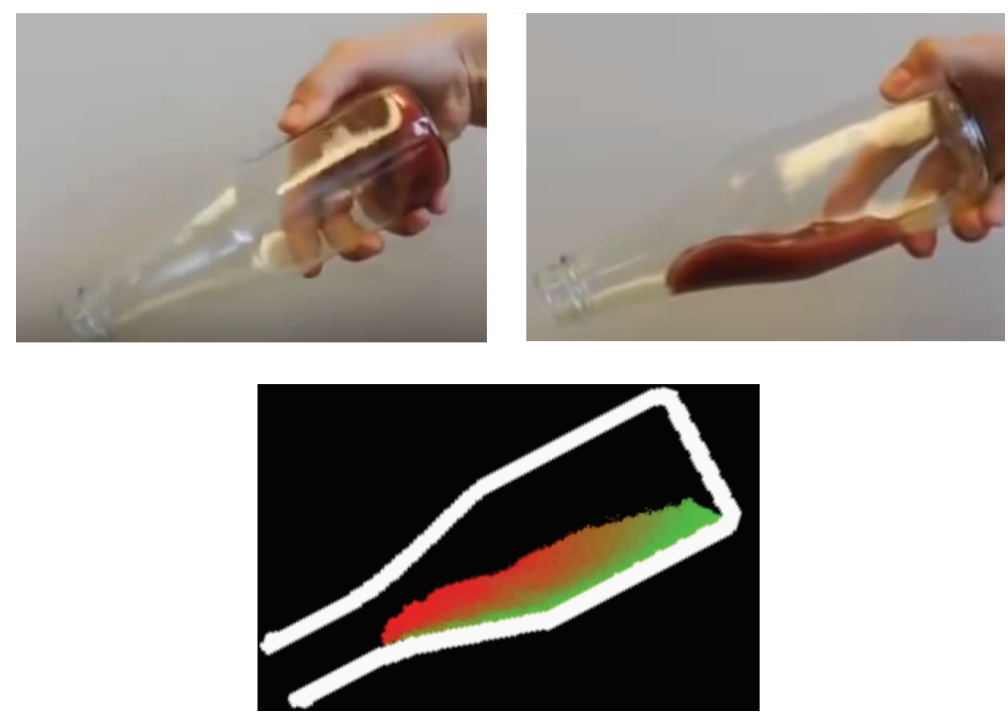

Figure 3. Hydrophilic vs. hydrophobic surfaces: no-slip vs. slip condition. Left: Classical ketchup flow. Right: Ketchup flowing in a bottle treated with a no-stick coating. Bottom: Numerical simulation (by Čanić and Wang) of flow with the Navier-slip boundary condition. The two snapshots were taken at the same time after the bottles had been tilted downwards. The colors in the third panel denote magnitude of fluid velocity: red is high, green is low velocity.

The benchmark problem defined on the domain shown in Figure 2, incorporating the Navier-slip condition as the kinematic coupling condition, can be summarized as follows: find $(\mathbf{u}, p, \boldsymbol{\eta})$ such that the following holds.

The fluid equations:

$$
\left.\begin{array}{rl}
\rho_{F}\left(\partial_{t} \mathbf{u}+\mathbf{u} \cdot \nabla \mathbf{u}\right) & =\nabla \cdot \boldsymbol{\sigma}(\mathbf{u}, p) \\
\nabla \cdot \mathbf{u} & =0
\end{array}\right\} \text { in } \Omega^{\eta}(t), t \in(0, T) .
$$


The elastic structure (Navier-slip coupling on $(0, L) \times(0, T)$ ):

$$
\begin{aligned}
\rho_{S} h \partial_{t t} \boldsymbol{\eta}(t, z)+\mathcal{L}_{e} \boldsymbol{\eta}(t, z) & =-J(t, z) \boldsymbol{\sigma}(\boldsymbol{\varphi}(t, z)) \mathbf{n}(t, z), \\
\partial_{t} \boldsymbol{\eta}(t, z) \cdot \mathbf{n}(t, z) & =\boldsymbol{u}(\boldsymbol{\varphi}(t, z)) \cdot \mathbf{n}(t, z), \\
\left(\partial_{t} \boldsymbol{\eta}(t, z)-\mathbf{u}(\boldsymbol{\varphi}(t, z)) \cdot \boldsymbol{\tau}(t, z)\right. & =\alpha \boldsymbol{\sigma}(\boldsymbol{\varphi}(t, z)) \mathbf{n}(t, z) \cdot \boldsymbol{\tau}(t, z),
\end{aligned}
$$

with

$$
\boldsymbol{\eta}(t, 0)=\partial_{z} \boldsymbol{\eta}(t, 0)=\boldsymbol{\eta}(t, L)=\partial_{z} \boldsymbol{\eta}(t, L)=0, t \in(0, T) .
$$

Boundary data at the inlet/outlet boundary $\Gamma_{\text {in/out }} \times(0, T)$ :

$$
p+\frac{\rho_{F}}{2}|\mathbf{u}|^{2}=P_{i}, \mathbf{u} \cdot \boldsymbol{\tau}=0 .
$$

Boundary data at the bottom, symmetry boundary $\Gamma_{b} \times(0, T)$ :

$$
\mathbf{u} \cdot \mathbf{n}=0, \partial_{\mathbf{n}} u_{\tau}=0,
$$

with $u_{\tau}$ denoting the tangental component of velocity $\mathbf{u}$.

\section{Initial conditions:}

$$
\mathbf{u}(0, .)=\mathbf{u}_{0}, \boldsymbol{\eta}(0, .)=\boldsymbol{\eta}_{0}, \partial_{t} \boldsymbol{\eta}(0, .)=\mathbf{v}_{0} .
$$

The following energy estimate holds:

$$
\begin{aligned}
& \frac{1}{2} \frac{d}{d t}\left(\rho_{F}\|\mathbf{u}\|_{L^{2}\left(\Omega^{\eta}(t)\right)}^{2}+\rho_{S} h\left\|\partial_{t} \boldsymbol{\eta}\right\|_{L^{2}(\Gamma)}^{2}+c\|\boldsymbol{\eta}\|_{\chi(\Gamma)}^{2}\right) \\
& \quad+\mu\|\mathbf{D}(\mathbf{u})\|_{L^{2}\left(\Omega^{\eta}(t)\right)}^{2}+\frac{1}{\alpha}\left\|u_{\tau}-\partial_{t} \eta_{\tau}\right\|_{L^{2}\left(\Gamma^{\eta}(t)\right)}^{2} \leq \mathbf{C},
\end{aligned}
$$

where $\mathbf{C}$ depends on the initial and boundary data, and the constant $c$ in front of the $\chi$-norm of $\boldsymbol{\eta}$ is associated with the coercivity of the structure operator $\mathcal{L}_{e}$. The reference configuration of the lateral boundary $\Gamma$ is $\Gamma=(0, L) \times\{R\}$.

The no-slip condition is reasonable for a great variety of problems for which the slip-length $\alpha$ is indeed very close to zero. However, in many cases of practical significance, no-slip is not adequate. Examples include flows over hydrophobic surfaces or surfaces treated with a no-stick coating (see Figure 3), flows over rough surfaces (such as those of, e.g., grooved vascular tissue scaffolds), and problems involving contact of smooth solids immersed in a viscous, incompressible fluid. More precisely, for flows over rough (rigid and fixed) surfaces, it has been shown that the Navier-slip boundary condition is the appropriate effective boundary condition [99, 100]. Instead of using the no-slip condition at the small groove scale, the effective Navier-slip boundary condition is applied at the corresponding groovefree, smooth boundary [99, 100]. Regarding contact of smooth bodies immersed in a viscous, incompressible fluid, recent studies have shown that contact is not possible if the no-slip boundary condition is considered [81,82,122]. A resolution to this no-collision paradox is to employ a different boundary condition, such as the Navier-slip boundary condition, which allows contact between smooth rigid bodies [12. Problems of this type arise, e.g., in modeling an elastic heart valve closure where different kinds of ad hoc gap conditions with the no-slip boundary condition have been used to get around this difficulty. 


\section{The arbitrary Lagrangian-Eulerian (ALE) Mappings}

To deal with the problems associated with the motion of the fluid domain, different approaches have been taken. One approach is to consider the entire moving boundary problem written in Lagrangian coordinates, as was done in [29, 30, 91. This is possible to do when the fluid domain is contained in a closed container and no fluid escapes the fluid domain, which is not the case with the benchmark problem considered above. Another approach is to map the problems from the moving domain onto a fixed reference domain using a family of mappings, known as the ALE mappings.

The ALE mappings are a family of (diffeomorphic) mappings, parameterized by $t$, such that

$$
\mathcal{A}^{\eta}(t): \Omega \rightarrow \Omega^{\eta}(t)
$$

ALE mappings have been extensively used in numerical simulations of moving boundary problems; see, e.g., [41,42,131. Recently, they have proven to be useful in mathematical analysis as well $[23,102,103$. In numerics, one of the reasons for the introduction of ALE mappings is the calculation of the discretized time derivative $\partial_{t} \mathbf{u}$ since a finite difference approximation of the time derivative (e.g., $\left.\left(\mathbf{u}^{n+1}-\mathbf{u}^{n}\right) / \Delta t\right)$ contains the functions $\mathbf{u}^{n+1}$ and $\mathbf{u}^{n}$ which are defined on two different domains, one corresponding to the time $t^{n+1}=(n+1) \Delta t$, and the other to $t^{n}=n \Delta t$. A way to calculate the time derivative of the fluid velocity is then to map the fluid velocities at times $t^{n+1}$ and $t^{n}$ onto a fixed domain $\Omega$ via the ALE mappings corresponding to $t^{n+1}$ and $t^{n}$, evaluate the time derivative there, and then map everything back to the physical domain $\Omega^{n}:=\Omega^{\eta^{n}}\left(t^{n}\right)$ to solve the fluid equations on the current domain $\Omega^{n}$. This introduces an extra advection term in the Navier-Stokes equations, describing the contribution due to the fluid domain motion, so that the Navier-Stokes equations in ALE form become

$$
\left.\begin{array}{rl}
\rho_{f}\left(\partial_{t} \mathbf{u}+\left(\left(\mathbf{u}-\mathbf{w}^{\eta^{n}}\right) \cdot \nabla\right) \mathbf{u}\right) & =\nabla \cdot \sigma \\
\nabla \cdot \mathbf{u} & =0
\end{array}\right\} \text { in }\left(t^{n}, t^{n+1}\right) \times \Omega^{n},
$$

where $\mathbf{w}^{\eta^{n}}=\partial_{t} \mathcal{A}^{\eta^{n}}\left(t^{n}\right)$ describes the fluid domain velocity, defined by the time derivative of the ALE mapping. In numerical solvers, the ALE mapping is often defined by the harmonic extension of the boundary data onto the fluid domain, i.e., as a solution to the elliptic problem,

$$
\begin{aligned}
\Delta \mathcal{A}^{\eta}(t) & =0 \text { on } \Omega, \\
\mathcal{A}^{\eta}(t) & =\eta(t) \text { on } \Gamma, \\
\mathcal{A}^{\eta}(t) & =0 \text { on } \partial \Omega \backslash \Gamma,
\end{aligned}
$$

that is calculated at every time step $t=t^{n}$. Other elliptic (elastic) operators have also been used.

In addition to numerical solvers, the ALE approach has recently been used to study the existence of solutions to moving boundary problems by either mapping the entire fluid problem onto the fixed domain $\Omega$ via an ALE mapping and analyzing the problem there (as in 102 104]) or by mimicking the approach described above, used in numerical simulations, and working on the current domain $\Omega^{n}$ (as in [23]). In the former case, an additional set of nonlinearities is introduced because the gradient operator $\nabla$ in physical space is mapped into an operator $\nabla^{\eta}$, defined on the fixed, reference domain. The mapped fluid velocity is no longer divergence-free 
in terms of the operator $\nabla$, which presents some difficulties when trying to use known results that hold for divergence-free functions.

Using ALE mappings in analysis requires assumptions on its regularity, which, of course, depends on the regularity of the fluid-structure interface $\eta$, not known a priori. In most works that use ALE mappings, an assumption on the regularity of the ALE mapping is made a priori, which is then justified a posteriori, after a proof showing existence of a solution with sufficient regularity of $\eta$ is obtained.

For the benchmark problem with no-slip coupling presented in section 2.1, one can introduce a family of ALE mappings, parameterized by $\eta$, given explicitly by

$$
\mathcal{A}^{\eta}(t): \Omega \rightarrow \Omega^{\eta}(t), \quad \mathcal{A}^{\eta}(t)(\tilde{z}, \tilde{r}):=\left(\begin{array}{c}
\tilde{z} \\
(R+\eta(t, \tilde{z})) \tilde{r}
\end{array}\right), \quad(\tilde{z}, \tilde{r}) \in \Omega,
$$

where $(\tilde{z}, \tilde{r})$ denote the coordinates in the reference domain $\Omega=(0, L) \times(0, R)$. Mapping $\mathcal{A}^{\eta}(t)$ is a bijection, and its Jacobian is given by

$$
\mathcal{J}_{\mathcal{A}^{\eta}}=\left|\operatorname{det} \nabla \mathcal{A}^{\eta}(t)\right|=|R+\eta(t, \tilde{z})| .
$$

Composite functions with the ALE mapping will be denoted by

$$
\mathbf{u}^{\eta}(t, .)=\mathbf{u}(t, .) \circ \mathcal{A}^{\eta}(t) \text { and } p^{\eta}(t, .)=p(t, .) \circ \mathcal{A}^{\eta}(t) .
$$

The derivatives of composite functions satisfy

$$
\partial_{t} \mathbf{u}=\partial_{t} \mathbf{u}^{\eta}-\left(\mathbf{w}^{\eta} \cdot \nabla^{\eta}\right) \mathbf{u}^{\eta}, \quad \nabla \mathbf{u}=\nabla^{\eta} \mathbf{u}^{\eta},
$$

where the ALE domain velocity, $\mathbf{w}^{\eta}$, and the transformed gradient, $\nabla^{\eta}$, are given by

$$
\mathbf{w}^{\eta}=\partial_{t} \eta \tilde{r} \mathbf{e}_{r}, \quad \nabla^{\eta}=\left(\begin{array}{c}
\partial_{\tilde{z}}-\tilde{r} \frac{\partial_{z} \eta}{R+\eta} \partial_{\tilde{r}} \\
\frac{1}{R+\eta} \partial_{\tilde{r}}
\end{array}\right)
$$

Note that

$$
\nabla^{\eta} \mathbf{v}=\nabla \mathbf{v}\left(\nabla A_{\eta}\right)^{-1}
$$

The following notation will also be useful:

$$
\sigma^{\eta}=-p^{\eta} \mathbf{I}+2 \mu \mathbf{D}^{\eta}\left(\mathbf{u}^{\eta}\right), \quad \mathbf{D}^{\eta}\left(\mathbf{u}^{\eta}\right)=\frac{1}{2}\left(\nabla^{\eta} \mathbf{u}^{\eta}+\left(\nabla^{\eta}\right)^{\tau} \mathbf{u}^{\eta}\right) .
$$

The resulting problem, defined entirely on the fixed, reference domain in the ALE framework, now reads find $\mathbf{u}(t, \tilde{z}, \tilde{r}), p(t, \tilde{z}, \tilde{r})$, and $\eta(t, \tilde{z})$ such that

$$
\begin{aligned}
& \left.\begin{array}{rl}
\rho_{f}\left(\partial_{t} \mathbf{u}^{\eta}+\left(\left(\mathbf{u}^{\eta}-\mathbf{w}^{\eta}\right) \cdot \nabla^{\eta}\right) \mathbf{u}^{\eta}\right) & =\nabla^{\eta} \cdot \boldsymbol{\sigma}^{\eta} \\
\nabla^{\eta} \cdot \mathbf{u}^{\eta} & =0
\end{array}\right\} \text { in }(0, T) \times \Omega, \\
& \left.\begin{array}{rl}
\mathbf{u}^{\eta} & =\partial_{t} \eta \mathbf{e}_{r} \\
\rho_{s} h \partial_{t}^{2} \eta+\mathcal{L}_{e} \eta & =-J \boldsymbol{\sigma}^{\eta} \mathbf{n} \cdot \mathbf{e}_{r}
\end{array}\right\} \text { on }(0, T) \times \Gamma, \\
& \left.\begin{array}{rl}
u_{r}^{\eta} & =0 \\
\partial_{r} u_{z}^{\eta} & =0
\end{array}\right\} \text { on }(0, T) \times \Gamma_{b}, \\
& \left.\begin{array}{rl}
p+\frac{\rho_{f}}{2}\left|\mathbf{u}^{\eta}\right|^{2} & =P_{\text {in } / \text { out }}(t) \\
u_{r}^{\eta} & =0
\end{array}\right\} \text { on }(0, T) \times \Gamma_{\text {in } / \text { out }}, \\
& \mathbf{u}^{\eta}(0, .)=\mathbf{u}_{0}^{\eta}, \eta(0, .)=\eta_{0}, \partial_{t} \eta(0, .)=v_{0} .
\end{aligned}
$$

A review of the recent result on existence theory related to this benchmark problem is presented next. 


\section{ReCEnt RESUlts AND OPEn PROBlems in FSI WITH ELASTIC STRUCTURES}

The development of existence theory for moving boundary, fluid-structure interaction problems started in the late 1990s/early 2000s. The first existence results were obtained for the cases in which the structure is completely immersed in the fluid and in which the structure was considered to be either a rigid body or described by a finite number of modal functions (see, e.g., [15, 31, 36, 37, 39, 47, 57, 122, and the references therein). The analysis of the coupling between the twodimensional or three-dimensional Navier-Stokes equations and two-dimensional or three-dimensional linear elasticity started in the early 2000s with works in which the coupling between the fluid and structure were assumed across a fixed fluid-structure interface (a linear coupling), as in [10, 11, 45, 93], and then extended to problems with nonlinear coupling in the works [14,25, 29, 30, 34, 35, 68, 70, 91, 95, 96, 102, 103 . More precisely, concerning nonlinear FSI models, the first FSI existence result, locally in time, was obtained in [14], where a strong solution for an interaction between an incompressible, viscous fluid in two dimensions and a one-dimensional viscoelastic string was obtained, assuming periodic boundary conditions. This result was extended by Lequeurre in [96, where the existence of a unique, local-intime, strong solution for any data and the existence of a global strong solution for small data were proved in the case when the structure is modeled as a clamped viscoelastic beam. Coutand and Shkoller proved existence, locally in time, of a unique, regular solution for an interaction between a viscous, incompressible fluid in three dimensions and a three-dimensional structure, immersed in the fluid, where the structure was modeled by the equations of linear [34] or quasi-linear [35] elasticity. In the case when the structure (solid) is modeled by a linear wave equation, Kukavica et al. proved the existence, locally in time, of a strong solution, assuming lower regularity for the initial data [88,91]. A similar result for compressible flows can be found in 92. In [116] Raymod et al. considered an FSI problem between a linear elastic solid immersed in an incompressible viscous fluid, and they proved the existence and uniqueness of a strong solution. Most of the above-mentioned existence results for strong solutions are local in time. In 89] a global existence result for small data was obtained by Ignatova et al. for a moving boundary FSI problem involving a damped linear wave equation with some additional damping terms in the coupling conditions, showing exponential decay in time of the solution. In the case when the structure is modeled as a two-dimensional elastic shell interacting with a viscous, incompressible fluid in three dimensions, the existence, locally in time, of a unique regular solution was proved by Shkoller et al. in [29, 30. We mention that the works of Shkoller et al. and Kukavica at al. were obtained in the context of Lagrangian coordinates, which were used for both the structure and fluid subproblems.

In the context of weak solutions, the first existence results came out in 2005 when Chambolle et al. showed the existence of a weak solution for an FSI problem between a three-dimensional incompressible, viscous fluid and a two-dimensional viscoelastic plate in [25]. Grandmont improved this result in 70] to hold for a two-dimensional elastic plate. A constructive existence proof for the interaction between an incompressible, viscous fluid and a linearly elastic Koiter shell with transverse displacement was designed in [102]. The first constructive existence proof for moving boundary problems was presented by Ladyzhenskaya in 1970, 
where the interaction between an incompressible, viscous fluid and a given moving boundary was constructed using a time-discretization approach, known as Rothe's method, assuming high regularity of the given interface 94. Muha and Canić designed a Rothe's-type method in the context of moving boundaries that are not known a priori in 2013 [102]. To deal with a moving boundary not known a priori, they introduced the time discretization via Lie operator splitting, which has been used in numerical schemes, as described in [66. The FSI problem studied in 102 is split into a fluid and a structure subproblem, with the coupling designed so that the resulting scheme is stable. This was achieved by using the results about the added mass effect, published in 24, which showed the importance of implicit treatment of the fluid and structure inertia in loosely coupled partitioned schemes. The splitting of the coupled problem in 102 was then done in such a way that the fluid and structure inertia terms are kept implicitly together, which provided a uniform energy estimate, not otherwise attainable using the classical DirichletNeumann partitioned schemes [115, 118. A compactness argument (discussed in section (5) was used to show that sub-sequence of approximate solutions converge to a weak solution to the coupled problem. After 2013 similar approaches were used to prove existence of a weak solution for a nonlinear FSI problem involving a nonlinear Koiter shell [105, a multi-layered structure [104, and a Koiter shell with the Navier-slip condition [106].

To complete the discussion of well-posedness, we mention here a result on continuous dependence of weak solutions on initial data, obtained in 79 for a fluid structure interaction problem with a free-boundary-type coupling condition.

In all these works the existence of a weak solution was proved for as long as the elastic boundary does not touch the bottom (rigid) portion of the fluid domain boundary. Recently, Grandmont and Hillairet showed that contact between a rigid bottom of a fluid container and a viscoelastic beam is not possible in finite time [71]. The finite-time contact involving thin and thick elastic structures interacting with an incompressible, viscous fluid is still open.

We conclude this section with a few general remarks related to the geometric nonlinearity in FSI problems for which the coupling across the current location of the moving interface is needed to describe the physical problem. The strong exchange of energy between the fluid and structure motion in the nonlinearly coupled problems gives rise to the various difficulties in the study of mathematical well-posedness. In particular, the functional spaces based on finite energy considerations may not provide sufficient regularity of the moving interface to even define the trace of the fluid velocity at the fluid-structure interface and, additionally, may lead to various fluid domain degeneracies; see Figure 4. These problems are particularly evident when elastic structures are thin (modeled by the reduced membrane or shell equations) and the structure model accounts for both transverse and tangential components of displacement, and the structure is interacting in three dimensions with the flow of an incompressible viscous fluid. In those cases the weak solution techniques based on finite energy spaces are often times insufficient to guarantee even the Lipschitz regularity of the fluid-structure interface; see [23]. This is one of the reasons why most literature on the existence of (weak) solutions to moving boundary problems involving thin elastic structures assumes only the transverse component of displacement to be different from zero [14,25, 68, 70, 95, 96, 102, 103. Recently, a three-dimensional FSI problem allowing transverse and tangential displacements of 


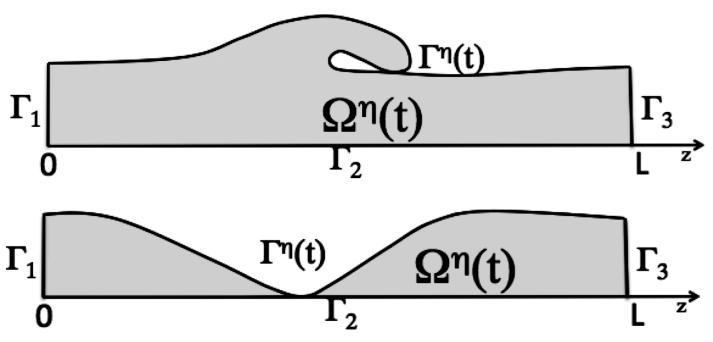

Figure 4. Two ways the fluid domain can degenerate. Top: Loss of injectivity of the ALE mapping $\mathcal{A}^{\eta}(t)$. Bottom: Loss of injectivity of the ALE mapping $\mathcal{A}^{\eta}(t)$ and loss of strict positivity of the Jacobian $\mathcal{A}^{\eta}(t)$.

a mesh-supported shell was studied in [23] where an existence of a weak solution in three dimensions was obtained under an extra assumption on the uniform Lipschitz property for the fluid-structure interface. FSI problems with elastic structures that are slightly more regular than the Koiter shell (such as, e.g., tripolar materials studied in [16,119]) do not suffer from this difficulty.

The issues related to nonzero transverse displacement cannot be avoided in FSI problems with Navier-slip coupling. This is the reason why the existence result in two dimensions for an FSI problem involving a Koiter shell interacting with the flow of an incompressible, viscous fluid via the Navier-slip condition [106] holds only until the fluid domain remains regular, in the sense that degeneracies of the type shown in Figure 4 do not occur. In problems with slip, compactness may be an additional problem since the regularizing effects by fluid viscosity are transferred to the structure only via the nonpenetration condition holding in the normal direction to the boundary. Nevertheless, the friction effects in the tangential direction can be used to compensate for the lack of regularization provided by the fluid viscosity. More details about compactness for problems on moving domains are presented next.

\section{Compactness}

Compactness results similar to the classical Aubin-Lions-Simon lemma [7, 124] that hold for moving boundary problems are difficult to obtain because, among other things, the function spaces depend on time via the fluid domain motion, and the fluid domains are not known a priori. A compactness result in generalized Bochner spaces $L^{2}(0, T ; H(t))$, where $H(t)$ is a family of Hilbert spaces which depend on time, is needed to deal with the fluid flow nonlinearities and with the geometric nonlinearities associated with the fluid domain motion. Such a compactness result should include conditions on the dynamic change of the fluid domain geometry that would contribute to the compactness argument.

To the best of our knowledge, there is no general compactness theory similar to the Aubin-Lions-Simon lemma [7, 124 for spaces $L^{2}(0, T ; H(t))$, where $H(t)$ depends on time. There are several compactness results for particular, specific problems for which the spatial domain depends on time, but they were proved using assumptions that hold for that particular problem at hand. The first result of this type was presented in Sather's PhD thesis [120] and then by Fujita and Sauer 
in [53], where they studied the Navier-Stokes equations in a given, noncylindrical domain, namely, in a domain which depends on time, and whose motion is given a priori. Similarly, in [33, Conca and San Martin used a compactness result to study a fluid-rigid body interaction problem, where an assumption on high regularity of the domain motion had to be used to obtain an existence result (see also [49, 122]). In the case of fluid-elastic structure interaction problems, the assumption on high regularity of the interface is typically not satisfied, thus different approaches need to be employed.

In the context of fluid-elastic structure interaction problems, we mention [39,70] where Desjardins, Esteban, Grandmont, and Le Tallec considered a fluid-elastic structure interaction problem between the flow of a viscous, incompressible fluid and an elastic/viscoelastic plate, in which a compactness argument based on Simon's theorem was used to show $L^{2}$-strong convergence of approximate solutions. We also mention 85. where this approach was used in the case of a non-Newtonian fluid. A similar problem, but in a more general geometrical setting, was studied in [95, where compactness of a set of approximate weak solutions, based on a particular linearization and regularization of the problem, was proved by using a modification of the ideas from the proof of Aubin-Lions lemma. Both approaches used the fluid viscosity and kinematic coupling condition to control high frequency oscillations of the structure velocity. Recently, a version of the Aubin-Lions lemma for a moving domain problem was proved in the context of compressible fluids (see [18]), where an existence of a solution to an FSI problem between a compressible fluid and a linearly elastic shell was obtained. The lack of the fluid incompressibility constraint simplifies the compactness argument for the velocity field.

Compactness results in more general frameworks were studied in [110,111], where Nägele, Rŭžička, and Lengeler developed a functional framework based on the flow method and the Piola transform for problems in smoothly moving domains, where the flow causing domain motion was given a priori. In those works a version of the Aubin-Lions lemma was obtained within this framework. A different version of the Aubin-Lions lemma, in a more general form, was also considered in [101]. The approach in [101] was based on negative Sobolev space-type estimates, defined on noncylindrical, i.e., time-dependent, domains. The latter approach did not require high degree of smoothness of the domain motion.

We also mention the results obtained in [12,28,101, where generalizations of the Aubin-Lions-Simon lemma in various types of nonlinear settings were obtained, as well as the work in [43, where a version of Aubin-Lions-Simon result was obtained in the context of finite-element spaces. We also mention the works by Elliott et al., where compactness arguments were developed and used to study parabolic problems on moving surfaces; see 26 .

Most of the works mentioned above were obtained for continuous time, i.e., the time variable was not discretized, and most of them were tailored for a particular application in mind. Working with discretized time brings some additional difficulties in terms of the uniform bounds for the time-shifts (translations in time). In the time-discretized case, namely, for the approaches based on Rothe's semidiscretization method, the uniform bounds on the time-shifts need to be somewhat stronger to guarantee compactness; see [44, Proposition 2]. In particular, the work in [44] addresses a version of the Aubin-Lions-Simon result for piecewise constant 
functions in time, which was obtained using Rothe's method but for a problem defined on a fixed Banach space.

The work presented in [107] concerns a generalization of the Aubin-Lions-Simon result which involves Hilbert spaces that are solution dependent and not necessarily known a priori. This is a significant step forward, since the result can be applied to a large class of moving boundary problems, including numerical solvers. To account for the time dependence of the function spaces associated with the motion of the fluid domains, the authors identified a new set of conditions, which quantify the dependence of the Hilbert spaces on time so that an extension of Aubin-LionsSimon result can be applied to a sequence of approximate solutions constructed using Rothe's method.

More precisely, the compactness result in [107 is designed for problems which can be described in general as evolution problems,

$$
\begin{aligned}
\frac{d \mathbf{u}}{d t} & =A^{t} \mathbf{u}, t \in(0, T), \\
\mathbf{u}(0) & =\mathbf{u}_{0},
\end{aligned}
$$

where $A^{t}: V(t) \rightarrow W(t)$ is a family of (nonlinear) spatial differential operators that depend on time as a parameter. For example, $\frac{d \mathbf{u}}{d t}=A^{t} \mathbf{u}$ may correspond to the Navier-Stokes equations for an incompressible, viscous fluid defined on a moving domain $\Omega(t)$. In this case, $A^{t}$ is a spatial differential operator that associates to each $\mathbf{u}$ the function $\nabla \cdot \boldsymbol{\sigma}-\mathbf{u} \cdot \nabla \mathbf{u}$, where $\boldsymbol{\sigma}$ is the fluid Cauchy stress tensor and $\mathbf{u}$ is divergence-free satisfying certain boundary conditions on $\Omega(t)$.

A way to "solve" this class of problems is to semi-discretize the problem in time by subdividing the time interval $(0, T)$ into $N$ subintervals of width $\Delta t=T / N$, and introduce the piecewise constant approximate functions,

$$
\mathbf{u}_{\Delta t}=\mathbf{u}_{\Delta t}^{n} \quad \text { for } t \in((n-1) \Delta t, n \Delta t], n=1, \ldots, N,
$$

which satisfy, e.g., a backwards Euler approximation of the problem on $\left(t^{n}, t^{n+1}\right)$,

$$
\frac{\mathbf{u}_{\Delta t}^{n+1}-\mathbf{u}_{\Delta t}^{n}}{\Delta t}=A^{t^{n+1}} \mathbf{u}_{\Delta t}^{n+1} \quad \text { or } \quad \frac{\mathbf{u}_{\Delta t}^{n+1}-\mathbf{u}_{\Delta t}^{n}}{\Delta t}=A^{t^{n}} \mathbf{u}_{\Delta t}^{n+1},
$$

where the choice of $A^{t^{n+1}}$ or $A^{t^{n}}$ depends on the problem at hand. For example, if the motion of the domain $\Omega(t)$ is specified a priori, $A^{t^{n+1}}$ is typically used, where $A^{t^{n+1}}$ describes an approximation of the spatial differential operator defined on the current domain $\Omega\left(t^{n+1}\right)$. If the motion of the domain $\Omega(t)$ is not known a priori but depends on the solution of the underlying problem, then $A^{t^{n}}$ is typically used, where $A^{t^{n}}$ describes an approximation of the spatial differential operator defined on the previous domain $\Omega\left(t^{n}\right)$.

Functions $\mathbf{u}_{\Delta t}$ are defined for all $t \in(0, T)$, and they are piecewise constant on the interval $((n-1) \Delta t, n \Delta t]$, where the constant is defined by its value at $n \Delta t$. This approach to solving the evolution problem (5.1) is sometimes called Rothe's method.

Rothe's method provides a constructive proof which uses semi-discretization of the continuous problem with respect to time to design approximate solutions $\left\{\mathbf{u}_{\Delta t}\right\}$ where $\Delta t=T / N$, for every $N \in \mathbb{N}$. It was first used by Ladyzhenskaya in 94, for a moving-boundary problem in which the motion of a smooth moving boundary was known a priori. The aim is to prove the existence of a sub-sequence of $\left\{\mathbf{u}_{\Delta t}\right\}$ which converges to a weak solution of (5.1) as $\Delta t \rightarrow 0$ or, equivalently, as $N \rightarrow \infty$. Since the problem is nonlinear, weak convergence is not sufficient to show that 
the limit is a weak solution of the underlying problem. This is why compactness arguments need to be employed to conclude that there exists a sub-sequence $\left\{\mathbf{u}_{\Delta t}\right\}$, which is precompact in a certain generalized Bochner space $L^{2}(0, T ; H(t))$. This will allow passage to the limit in nonlinear terms and will show that the limit, as $\Delta t \rightarrow 0$, of approximate weak solutions satisfies the weak formulation of the continuous problem.

Employing this strategy to prove the existence of weak solutions to this class of problems is highly nontrivial, and it is at the center of the current research in this area 102, 104 106. The main source of difficulties is associated with the fact that for every $N \in \mathbb{N}$ and $n \in\{1, \ldots, N\}$, the approximate weak solutions $\mathbf{u}_{\Delta t}^{n}$, which are functions of the spatial variables, belong to different solution spaces $V_{\Delta t}^{n}$, which are associated with the operators $A^{t^{n}}: V_{\Delta t}^{n} \rightarrow W_{\Delta t}^{n}$, and which are defined on different domains $\Omega\left(t_{\Delta t}^{n}\right)$, thus $V_{\Delta t}^{n}=V\left(\Omega\left(t_{\Delta t}^{n}\right)\right)$. We would like to find the conditions under which $\left\{\mathbf{u}_{\Delta t}\right\}$ is precompact in some $L^{2}\left(0, T ; H\left(\Omega_{\Delta t}(t)\right)\right)$, where the definition of $L^{2}\left(0, T ; H\left(\Omega_{\Delta t}(t)\right)\right)$ needs to be made precise. Namely, we want to find the conditions under which there exists a sub-sequence, also denoted by $\left\{\mathbf{u}_{\Delta t}\right\}$, which converges in $L^{2}\left(0, T ; H\left(\Omega_{\Delta t}(t)\right)\right)$ to a function in $L^{2}(0, T ; H(\Omega(t)))$, as $\Delta t \rightarrow 0$.

There are two ways to make the notion of convergence in $L^{2}\left(0, T ; H\left(\Omega_{\Delta t}(t)\right)\right)$ precise. One way is to introduce a family of mappings, which map the domains $\Omega\left(t_{\Delta t}^{n}\right)$ onto a fixed domain $\Omega$ and work in the space $L^{2}(0, T ; H(\Omega))$. The other approach is to extend the functions $\mathbf{u}_{\Delta t}^{n}$ onto a larger, fixed domain $\Omega^{M}$ and work in the space $L^{2}\left(0, T ; H\left(\Omega^{M}\right)\right)$. In both cases, certain conditions describing the regularity in time of the domain motion need to be satisfied, in order for a compactness argument to hold. In 107 those conditions are identified, and a generalization of the Aubin-Lions-Simon compactness result was obtained, which can be used in both approaches, mentioned above.

The compactness result from [107. was applied to study existence of solutions to FSI with Koiter shell [102, 105, to FSI involving multi-layered structures [104], and to FSI with Koiter shell and Navier-slip coupling [106. Since the result is based on the backwards Euler time discretization approaches to the coupled FSI problem, the compactness result from [107] is a promising tool for proving convergence of numerical schemes that use the backwards Euler scheme to discretize the problem in time; see [19]22].

\section{FSI WITH RIGID BODIES}

The study of the motion of rigid bodies immersed in an incompressible, viscous fluid started almost 50 years ago with the works of Weinberger [130] and Sauer [121, who investigated the stationary problems. The study continued by the works of Galdi et al. who studied both the stationary and dynamic problems [58 61], that paved the way for the most recent results in this field which we survey below. Before we give a more detailed account of recent developments, we first present a mathematical formulation of a benchmark problem in fluid-structure interaction between a rigid body and an incompressible, viscous fluid.

6.1. FSI benchmark problem with rigid bodies. We consider an FSI problem between an incompressible, viscous fluid and a motion of a solid in a fluid container; see Figure 5 


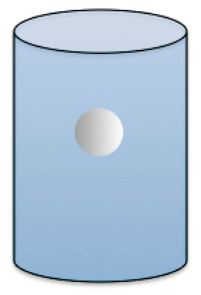

FIGURE 5. A sketch of a falling rigid ball in a fluid container.

We denote by $\Omega$ the region corresponding to the fluid container and by $S_{0} \subset \Omega$ the solid (rigid structure) in the container with the center of mass $\boldsymbol{q}_{0}$. The motion of a rigid body is fully described by the translation of its center of mass, and by the (rigid) rotation about the center of mass, described by the functions

$$
\boldsymbol{q}:[0, T] \rightarrow \mathbb{R}^{3} \quad \text { and } \quad \boldsymbol{Q}:[0, T] \rightarrow S O(3),
$$

where $S O(3)$ is the group of rotations in three dimensions, and $\boldsymbol{q}$ describes the translation of the center of mass. The trajectory of all the points in the rigid body is described by

$$
\boldsymbol{x}=\boldsymbol{B}(t, \boldsymbol{y})=\boldsymbol{q}(t)+\boldsymbol{Q}(t)(\boldsymbol{y}-\boldsymbol{q}(0)), \boldsymbol{y} \in S_{0}, t \in[0, T] .
$$

At time $t$, the solid occupies the set

$$
S(t)=\left\{\boldsymbol{x} \in \Omega_{0} \mid \boldsymbol{x}=\boldsymbol{B}(t, \boldsymbol{y}), \boldsymbol{y} \in S_{0}=\boldsymbol{B}\left(t, S_{0}\right)\right\},
$$

defining the fluid domain at time $t, \Omega_{F}(t)=\Omega \backslash \overline{S(t)}$. As in the case of elastic structures, the fluid domain is not known a priori since it depends on the structural unknowns in the problem, namely the location of the rigid solid at time $t$.

As before, the fluid flow is described by Navier-Stokes equations for an incompressible, viscous Newtonian fluid (2.1), while the equations of motion of the rigid body are given by a system of six ordinary differential equations (Euler equations) describing the conservation of linear and angular momentum,

$$
\begin{aligned}
& m \frac{d^{2} \boldsymbol{q}}{d t^{2}}=\boldsymbol{f}, \\
& \frac{d(\boldsymbol{J} \boldsymbol{\omega})}{d t}=\boldsymbol{g},
\end{aligned}
$$

where $m$ is the mass of the rigid body, $\boldsymbol{\omega}$ corresponds to angular velocity, $\boldsymbol{J}$ is the inertial tensor, and $\boldsymbol{f}$ and $\boldsymbol{g}$ are the total force and torque acting on the rigid body, respectively. The inertial tensor is defined by

$$
\boldsymbol{J}=\int_{S(t)} \rho_{S}\left(|\boldsymbol{x}-\boldsymbol{q}(t)|^{2} \boldsymbol{I}-(\boldsymbol{x}-\boldsymbol{q}(t)) \otimes(\boldsymbol{x}-\boldsymbol{q}(t))\right) d \boldsymbol{x},
$$

where $\rho_{S}$ is the structure density.

The coupling. The fluid and structure are coupled through two sets of coupling conditions: the kinematic and dynamics coupling conditions. For the kinematic coupling condition we take the no-slip condition, which says that the trace of the fluid velocity $\mathbf{u}$ at the rigid body boundary is equal to the velocity $\boldsymbol{u}_{S}$ of the rigid body itself,

$$
\mathbf{u}(t, \boldsymbol{x})=\boldsymbol{u}_{S}(t, \boldsymbol{x}), \boldsymbol{x} \in \partial S(t), t \in(0, T),
$$


where $\boldsymbol{u}_{S}$ is given by

$$
\boldsymbol{u}_{S}=\frac{d \boldsymbol{x}}{d t}=\frac{d}{d t}(\boldsymbol{q}(t)+\boldsymbol{Q}(t)(\boldsymbol{y}-\boldsymbol{q}(0)))=\boldsymbol{q}^{\prime}(t)+\boldsymbol{\omega}(t) \times(\boldsymbol{y}-\boldsymbol{q}(0)) .
$$

The dynamic coupling condition describes the balance of forces and torque. It says that the motion of the rigid structure in the fluid is driven by the contact force exerted by the fluid onto the structure. More precisely, the force and torque $f$ and $\boldsymbol{g}$ in (6.1) are replaced by

$$
\boldsymbol{f}=-\int_{\partial S(t)} \boldsymbol{\sigma} \mathbf{n} d s(\boldsymbol{x}), \quad \boldsymbol{g}=-\int_{\partial S(t)}(\boldsymbol{x}-\boldsymbol{q}(t)) \times \boldsymbol{\sigma} \mathbf{n} d s(\boldsymbol{x}) .
$$

Thus, the benchmark nonlinear moving-boundary problem, describing fluid-structure interaction between an incompressible, viscous fluid and a rigid solid immersed in the fluid, can be summarized as follows. Find $(\mathbf{u}, p, \boldsymbol{q}, \boldsymbol{\omega})$ such that

$$
\begin{aligned}
& \left.\begin{array}{rl}
\rho_{f}\left(\partial_{t} \mathbf{u}+(\mathbf{u} \cdot \nabla) \mathbf{u}\right) & =\nabla \cdot \boldsymbol{\sigma}(\mathbf{u}, p) \\
\nabla \cdot \mathbf{u} & =0
\end{array}\right\} \text { in } \Omega_{F}(t), t \in(0, T), \\
& \mathbf{u}=\boldsymbol{q}^{\prime}(t)+\boldsymbol{\omega}(t) \times(\boldsymbol{x}-\boldsymbol{q}(0)) \text { on }(0, T) \times \partial S(t), \\
& \left.\begin{array}{rl}
m \frac{d^{2} \boldsymbol{q}}{d t^{2}} & =-\int_{\partial S(t)} \boldsymbol{\sigma} \mathbf{n} d s(\boldsymbol{x}) \\
\frac{d(\boldsymbol{J} \boldsymbol{\omega})}{d t} & =-\int_{\partial S(t)}(\boldsymbol{x}-\boldsymbol{q}(t)) \times \boldsymbol{\sigma} \mathbf{n} d s(\boldsymbol{x})
\end{array}\right\} \text { in }(0, T), \\
& \mathbf{u}=\mathbf{0} \text { on } \partial \Omega, \\
& \mathbf{u}(0, .)=\mathbf{u}_{0}, \text { in } \Omega, \boldsymbol{q}(0, .)=\boldsymbol{q}_{0}, \boldsymbol{q}^{\prime}(0, .)=\boldsymbol{a}_{0}, \boldsymbol{\omega}(0, .)=\boldsymbol{\omega}_{0} .
\end{aligned}
$$

Weak solutions. A weak solution is a function $(\mathbf{u}, \boldsymbol{B})$ which satisfies the following two conditions.

1. The function $\boldsymbol{B}(t, \cdot): \mathbb{R}^{3} \rightarrow \mathbb{R}^{3}$, which defines the time-dependent set $S(t)=$ $\boldsymbol{B}(t, S)$ and the corresponding Eulerian velocity $\boldsymbol{u}_{S}$, is an orientation preserving isometry.

2. The function $\mathbf{u} \in L^{2}(0, T ; V(t)) \cap L^{\infty}\left(0, T ; L^{2}(\Omega)\right.$ satisfies

$$
\begin{aligned}
\int_{0}^{T} \int_{\Omega \backslash \partial S(t)} & \left\{\mathbf{u} \cdot \partial_{t} \boldsymbol{\psi}+(\mathbf{u} \otimes \mathbf{u}): \mathbf{D}(\mathbf{u}): \mathbf{D}(\boldsymbol{\psi})\right\} d \boldsymbol{x} d t-\int_{\Omega} \mathbf{u}(T) \boldsymbol{\psi}(T) d \boldsymbol{x} \\
& =-\int_{\Omega} \mathbf{u}_{0} \boldsymbol{\psi}(0) d \boldsymbol{x}
\end{aligned}
$$

for all test functions $\boldsymbol{\psi} \in H^{2}(0, T ; V(t))$, where

$$
V(t)=\left\{\mathbf{v} \in H_{0}^{1}(\Omega) \mid \operatorname{div} \mathbf{v}=0, \mathbf{D}(\mathbf{v})=0 \text { in } S(t)\right\} .
$$

\section{RECENT RESUlts AND OPEN PROBLEMS IN FSI WITH RIGID SOLIDS}

Existence of a unique, local-in-time (or small data) strong solution is now known in both two and three space dimensions and for both the slip [1,128, and the no-slip coupling 36, 67, 97, 126.

In terms of weak solutions of Leray-Hopf type, existence up to collision was obtained by Gérard-Varet and Hillairet in 64 for the slip coupling, and more recently by Chemetov and Nečasová in [26], where they showed global-in-time existence including collision, assuming the Navier-slip condition prescribed at the solid 
boundary, and no-slip condition at the container boundary. Global existence with the no-slip coupling was established in [32, 37, 38, 80,122 .

The question of uniqueness of the weak solution is still largely open. Even for the classical case of the three-dimensional Navier-Stokes equations, the uniqueness of the Leray-Hopf weak solution is an outstanding open problem (see, e.g., [56]). However, there are classical results of weak-strong uniqueness type (see, e.g., 56, 123, 127]) which state that the strong solution (defined in an appropriate way) is unique in a larger class of weak solutions. For the Navier-Stokes equations the weak solutions that satisfy Serrin's conditions are regular [123. In the most recent paper by Muha, Nečasová, and Radošević [108], these classical weak-strong uniqueness type results are extended to the case of a fluid-rigid body system under the condition that the rigid body does not touch the boundary of the container. Namely, in the case of contact it has been shown that weak solutions are not unique (see [48,125]) because there are multiple ways of extending the solution beyond the contact.

While these results discuss uniqueness of strong solutions, the results on uniqueness of weak solutions are sparse. The principal difficulty lies in the fact that different solutions are defined on different domains, so comparison between solutions is difficult. Usually, to handle this difficulty, the problem is mapped onto a fixed domain using a mapping that depends on the regularity of solutions, so strong solutions are easier to deal with. In 2015, however, uniqueness of weak solution for a fluid-rigid body system in the two-dimensional case was obtained by Glass and Sueur in 65. for the no-slip case, and by Bravin in 2019 for the slip case [17, while uniqueness results of weak-strong type were recently published in [27, 40, 54. In [40] the authors studied a rigid body with its cavity filled with fluid, while in [27] a rather high regularity for strong solutions was required for the uniqueness result to hold (the time derivative and second spatial derivatives of the fluid velocity were required to be in $L^{2}$ ). In [54 Galdi, Mácha, and Nečasová studied a rigid body with its cavity filled with a compressible fluid and showed a weak-strong uniqueness property using a relative entropy inequality. The most resent result by Muha, Nečasová, and Radošević [108] generalizes these results, since they prove, for both the slip and no-slip cases, a generalization of the well-known weak-strong uniqueness result for the Navier-Stokes equations to the fluid-rigid body system. More precisely, they prove that weak solutions which additionally satisfy the Prodi-Serrin $L^{r}-L^{s}$ condition are unique in the class of Leray-Hopf weak solutions.

\section{Finite-Time CONTACT}

As already addressed in the works related to global-in-time existence of weak solutions mentioned above, global existence of solutions to FSI problems involving incompressible, viscous fluids is affected by the possibility of contact: either contact between rigid bodies immersed in the fluid, contact between elastic structures immersed in the fluid, or contact between an elastic structure with the fluid container (rigid) boundary. While in the case of compressible fluids, contact of rigid bodies is possible in finite time [47, the incompressible, viscous case is different since contact in finite time between rigid bodies is not possible for the scenarios studied in $32,62,64,72,80,83,98$. In a pioneering work in 2009 Nestupa and Penel showed that contact in finite time between rigid bodies immersed in a viscous, incompressible fluid is possible if the Navier-slip boundary condition is used, which 
became a precursor for a number of existence results involving a slip condition and FSI with rigid solids, described above.

Finite-time contact involving elastic structures remains an outstanding open problem, although a result from 2016 by Grandmont and Hillairet [1], indicates that finite-time contact with the no-slip condition and deformable structure is impossible. More precisely, Grandmont and Hillairet studied the interaction between a one-dimensional viscoelastic beam and a two-dimensional viscous, incompressible fluid, assuming no-slip coupling, and showed (1) that contact in finite time is not possible, and (2) that strong solutions exist globally in time. Their result is the first no-contact result involving deformable solids, and the first global existence result for FSI problems with an incompressible, viscous fluid and deformable structures.

\section{About THE AUthor}

Sunčica Čanić is professor of mathematics at University of California, Berkeley, and she is currently the Miller Research Professor at the Miller Institute, Berkeley. Her expertise is in partial differential equations and applications of partial differential equations in biology and medicine.

\section{REFERENCES}

[1] H. Al Baba, N. V. Chemetov, Š. Nečasová, and B. Muha, Strong solutions in $L^{2}$ framework for fluid-rigid body interaction problem. Mixed case, Topol. Methods Nonlinear Anal. 52 (2018), no. 1, 337-350, DOI 10.12775/tmna.2018.028. MR3867991

[2] A. Alphonse and C. M. Elliott, A Stefan problem on an evolving surface, Philos. Trans. Roy. Soc. A 373 (2015), no. 2050, 20140279, 16, DOI 10.1098/rsta.2014.0279. MR.3393311

[3] A. Alphonse and C. M. Elliott, Well-posedness of a fractional porous medium equation on an evolving surface, Nonlinear Anal. 137 (2016), 3-42, DOI 10.1016/j.na.2016.01.010. MR.3485116

[4] A. Alphonse, C. M. Elliott, and B. Stinner, An abstract framework for parabolic PDEs on evolving spaces, Port. Math. 72 (2015), no. 1, 1-46, DOI 10.4171/PM/1955. MR3323509

[5] A. Alphonse, C. M. Elliott, and B. Stinner, On some linear parabolic PDEs on moving hypersurfaces, Interfaces Free Bound. 17 (2015), no. 2, 157-187, DOI 10.4171/IFB/338. MR.3391968

[6] A. Alphonse, C. M. Elliott, and J. Terra, A coupled ligand-receptor bulk-surface system on a moving domain: well posedness, regularity, and convergence to equilibrium, SIAM J. Math. Anal. 50 (2018), no. 2, 1544-1592, DOI 10.1137/16M110808X. MR3775132

[7] J.-P. Aubin, Un théorème de compacité (French), C. R. Acad. Sci. Paris 256 (1963), 50425044. MR 152860

[8] M. Astorino, J.-F. Gerbeau, O. Pantz, and K.-F. Traoré, Fluid-structure interaction and multi-body contact: application to aortic valves, Comput. Methods Appl. Mech. Engrg. 198 (2009), no. 45-46, 3603-3612, DOI 10.1016/j.cma.2008.09.012. MR2571824

[9] F. P. T. Baaijens, A fictitious domain/mortar element method for fluid-structure interaction, Internat. J. Numer. Methods Fluids 35 (2001), no. 7, 743-761, DOI 10.1002/fld.153. MR 1826849

[10] V. Barbu, Z. Grujić, I. Lasiecka, and A. Tuffaha, Existence of the energy-level weak solutions for a nonlinear fluid-structure interaction model, Fluids and waves, Contemp. Math., vol. 440, Amer. Math. Soc., Providence, RI, 2007, pp. 55-82, DOI 10.1090/conm/440/08476. MR.2359449

[11] V. Barbu, Z. Grujić, I. Lasiecka, and A. Tuffaha, Smoothness of weak solutions to a nonlinear fluid-structure interaction model, Indiana Univ. Math. J. 57 (2008), no. 3, 1173-1207, DOI 10.1512/iumj.2008.57.3284. MR.2429089

[12] J. W. Barrett and E. Süli, Reflections on Dubinski乞̌'s nonlinear compact embedding theorem, Publ. Inst. Math. (Beograd) (N.S.) 91(105) (2012), 95-110, DOI 10.2298/PIM1205095B. MR2963813 
[13] S. Basting, A. Quaini, S. Čanić, and R. Glowinski, Extended ALE method for fluid-structure interaction problems with large structural displacements, J. Comput. Phys. 331 (2017), 312336, DOI 10.1016/j.jcp.2016.11.043. MR3588694

[14] H. Beirão da Veiga, On the existence of strong solutions to a coupled fluid-structure evolution problem, J. Math. Fluid Mech. 6 (2004), no. 1, 21-52, DOI 10.1007/s00021-003-0082-5. MR.2027753

[15] M. Boulakia, Existence of weak solutions for the motion of an elastic structure in an incompressible viscous fluid (English, with English and French summaries), C. R. Math. Acad. Sci. Paris 336 (2003), no. 12, 985-990, DOI 10.1016/S1631-073X(03)00235-8. MR1993967

[16] M. Boulakia, Existence of weak solutions for an interaction problem between an elastic structure and a compressible viscous fluid (English, with English and French summaries), J. Math. Pures Appl. (9) 84 (2005), no. 11, 1515-1554, DOI 10.1016/j.matpur.2005.08.004. MR2181459

[17] M. Bravin, Energy equality and uniqueness of weak solutions of a "viscous incompressible fluid + rigid body" system with Navier slip-with-friction conditions in a $2 D$ bounded domain, J. Math. Fluid Mech. 21 (2019), no. 2, Paper No. 23, 31, DOI 10.1007/s00021-019-0425-6. MR 3928692

[18] D. Breit and S. Schwarzacher, Compressible fluids interacting with a linear-elastic shell, Arch. Ration. Mech. Anal. 228 (2018), no. 2, 495-562, DOI 10.1007/s00205-017-1199-8. MR 3766983

[19] M. Bukač and S. Čanić, Longitudinal displacement in viscoelastic arteries: a novel fluidstructure interaction computational model, and experimental validation, Math. Biosci. Eng. 10 (2013), no. 2, 295-318, DOI 10.3934/mbe.2013.10.295. MR.3019434

[20] M. Bukač, S. Čanić, R. Glowinski, B. Muha, and A. Quaini, A modular, operator-splitting scheme for fluid-structure interaction problems with thick structures, Internat. J. Numer. Methods Fluids 74 (2014), no. 8, 577-604, DOI 10.1002/fld.3863. MR3168786

[21] M. Bukač, S. Čanić, R. Glowinski, J. Tambača, and A. Quaini, Fluid-structure interaction in blood flow capturing non-zero longitudinal structure displacement, J. Comput. Phys. 235 (2013), 515-541, DOI 10.1016/j.jcp.2012.08.033. MR3017610

[22] M. Bukač, S. Čanić, and B. Muha, A partitioned scheme for fluid-composite structure interaction problems, J. Comput. Phys. 281 (2015), 493-517, DOI 10.1016/j.jcp.2014.10.045. MR.3281984

[23] S. Canić, M. Galić, and B. Muha. Analysis of a nonlinear moving-boundary, 3D fluid-meshshell interaction problem, arXiv:2940144 (submitted 2019).

[24] P. Causin, J. F. Gerbeau, and F. Nobile, Added-mass effect in the design of partitioned algorithms for fluid-structure problems, Comput. Methods Appl. Mech. Engrg. 194 (2005), no. 42-44, 4506-4527, DOI 10.1016/j.cma.2004.12.005. MR.2157973

[25] A. Chambolle, B. Desjardins, M. J. Esteban, and C. Grandmont, Existence of weak solutions for the unsteady interaction of a viscous fluid with an elastic plate, J. Math. Fluid Mech. 7 (2005), no. 3, 368-404, DOI 10.1007/s00021-004-0121-y. MR2166981

[26] N. V. Chemetov and Š. Nečasová, The motion of the rigid body in the viscous fluid including collisions. Global solvability result, Nonlinear Anal. Real World Appl. 34 (2017), 416-445, DOI 10.1016/j.nonrwa.2016.09.011. MR.3567970

[27] N. V. Chemetov, Š. Nečasová, and B. Muha, Weak-strong uniqueness for fluid-rigid body interaction problem with slip boundary condition, J. Math. Phys. 60 (2019), no. 1, 011505, 13, DOI 10.1063/1.5007824. MR3896559

[28] X. Chen, A. Jüngel, and J.-G. Liu, A note on Aubin-Lions-Dubinskiǔ lemmas, Acta Appl. Math. 133 (2014), 33-43, DOI 10.1007/s10440-013-9858-8. MR.3255076

[29] C. H. A. Cheng, D. Coutand, and S. Shkoller, Navier-Stokes equations interacting with a nonlinear elastic biofluid shell, SIAM J. Math. Anal. 39 (2007), no. 3, 742-800, DOI 10.1137/060656085. MR2349865

[30] C. H. A. Cheng and S. Shkoller, The interaction of the 3D Navier-Stokes equations with a moving nonlinear Koiter elastic shell, SIAM J. Math. Anal. 42 (2010), no. 3, 1094-1155, DOI $10.1137 / 080741628$. MR.2644917

[31] C. Conca, J. San Martín H., and M. Tucsnak, Motion of a rigid body in a viscous fluid (English, with English and French summaries), C. R. Acad. Sci. Paris Sér. I Math. 328 (1999), no. 6, 473-478, DOI 10.1016/S0764-4442(99)80193-1. MR1680008 
[32] C. Conca, J. San Martín H., and M. Tucsnak, Existence of solutions for the equations modelling the motion of a rigid body in a viscous fluid, Comm. Partial Differential Equations 25 (2000), no. 5-6, 1019-1042, DOI 10.1080/03605300008821540. MR.1759801

[33] C. Conca and J. San Martín H., Existence of solutions for the equations modelling the motion of a rigid body in a viscous fluid, Comm. Partial Differential Equations 25 (2000), no. 5-6, 99-110.

[34] D. Coutand and S. Shkoller, Motion of an elastic solid inside an incompressible viscous fluid, Arch. Ration. Mech. Anal. 176 (2005), no. 1, 25-102, DOI 10.1007/s00205-004-03407. MR2185858

[35] D. Coutand and S. Shkoller, The interaction between quasilinear elastodynamics and the Navier-Stokes equations, Arch. Ration. Mech. Anal. 179 (2006), no. 3, 303-352, DOI 10.1007/s00205-005-0385-2. MR2208319

[36] P. Cumsille and T. Takahashi, Well-posedness for the system modelling the motion of a rigid body of arbitrary form in an incompressible viscous fluid, Czechoslovak Math. J. 58(133) (2008), no. 4, 961-992, DOI 10.1007/s10587-008-0063-2. MR2471160

[37] B. Desjardins and M. J. Esteban, Existence of weak solutions for the motion of rigid bodies in a viscous fluid, Arch. Ration. Mech. Anal. 146 (1999), no. 1, 59-71, DOI 10.1007/s002050050136. MR.1682663

[38] B. Desjardins and M. J. Esteban, On weak solutions for fluid-rigid structure interaction: compressible and incompressible models, Comm. Partial Differential Equations 25 (2000), no. 7-8, 1399-1413, DOI 10.1080/03605300008821553. MR.1765138

[39] B. Desjardins, M. J. Esteban, C. Grandmont, and P. Le Tallec, Weak solutions for a fluidelastic structure interaction model, Rev. Mat. Complut. 14 (2001), no. 2, 523-538, DOI 10.5209/rev_REMA.2001.v14.n2.17030. MR.1871311

[40] K. Disser, G. P. Galdi, G. Mazzone, and P. Zunino, Inertial motions of a rigid body with a cavity filled with a viscous liquid, Arch. Ration. Mech. Anal. 221 (2016), no. 1, 487-526, DOI 10.1007/s00205-016-0966-2. MR3483900

[41] J. Donea, Arbitrary Lagrangian-Eulerian finite element methods, in: Computational methods for transient analysis, North-Holland, Amsterdam,1983.

[42] J. Donea, A. Huerta, J. P. Ponthot, and A. Rodriguez-Ferran, Arbitrary Lagrangian-Eulerian Method, Encyclopedia of Computational Mathematics, Wiley, 2004.

[43] J. Droniou, R. Eymard, T. Gallouët, C. Guichard, and R. Herbin, The gradient discretisation method, 2016.

[44] M. Dreher and A. Jüngel, Compact families of piecewise constant functions in $L^{p}(0, T ; B)$, Nonlinear Anal. 75 (2012), no. 6, 3072-3077, DOI 10.1016/j.na.2011.12.004. MR2890969

[45] Q. Du, M. D. Gunzburger, L. S. Hou, and J. Lee, Analysis of a linear fluidstructure interaction problem, Discrete Contin. Dyn. Syst. 9 (2003), no. 3, 633-650, DOI 10.3934/dcds.2003.9.633. MR.1974530

[46] L. J. Fauci and R. Dillon, Biofluidmechanics of reproduction, Annu. Rev. Fluid Mech., vol. 38, Annual Reviews, Palo Alto, CA, 2006, pp. 371-394, DOI 10.1146/annurev.fluid.37.061903.175725. MR2206979

[47] E. Feireisl, On the motion of rigid bodies in a viscous compressible fluid, Arch. Ration. Mech. Anal. 167 (2003), no. 4, 281-308, DOI 10.1007/s00205-002-0242-5. MR.1981859

[48] E. Feireisl, On the motion of rigid bodies in a viscous fluid: Mathematical theory in fluid mechanics (Paseky, 2001), Appl. Math. 47 (2002), no. 6, 463-484, DOI 10.1023/A:1023245704966. MR.1948192

[49] E. Feireisl, M. Hillairet, and Š. Nečasová, On the motion of several rigid bodies in an incompressible non-Newtonian fluid, Nonlinearity 21 (2008), no. 6, 1349-1366, DOI 10.1088/0951$7715 / 21 / 6 / 012$. MR2422384

[50] M. A. Fernández, Incremental displacement-correction schemes for incompressible fluidstructure interaction, Numer. Math. 123 (2013), no. 1, 21-65, DOI 10.1007/s00211-0120481-9. MR 3004589

[51] M. A. Fernández, J.-F. Gerbeau, and C. Grandmont, A projection semi-implicit scheme for the coupling of an elastic structure with an incompressible fluid, Internat. J. Numer. Methods Engrg. 69 (2007), no. 4, 794-821, DOI 10.1002/nme.1792. MR2284413

[52] C. A. Figueroa, I. E. Vignon-Clementel, K. E. Jansen, T. J. R. Hughes, and C. A. Taylor, A coupled momentum method for modeling blood flow in three-dimensional deformable 
arteries, Comput. Methods Appl. Mech. Engrg. 195 (2006), no. 41-43, 5685-5706, DOI 10.1016/j.cma.2005.11.011. MR2243323

[53] H. Fujita and N. Sauer, On existence of weak solutions of the Navier-Stokes equations in regions with moving boundaries, J. Fac. Sci. Univ. Tokyo Sect. I 17 (1970), 403-420. MR298258

[54] G. P. Galdi, V. Mácha, and Š. Nečasová, On weak solutions to the problem of a rigid body with a cavity filled with a compressible fluid, and their asymptotic behavior, arXiv:1903.01453 (2019).

[55] G. P. Galdi, An introduction to the mathematical theory of the Navier-Stokes equations. Vol. I, Springer Tracts in Natural Philosophy, vol. 38, Springer-Verlag, New York, 1994. Linearized steady problems. MR 1284205

[56] G. P. Galdi, An introduction to the Navier-Stokes initial-boundary value problem, Fundamental directions in mathematical fluid mechanics, Adv. Math. Fluid Mech., Birkhäuser, Basel, 2000, pp. 1-70. MR 1798753

[57] G. P. Galdi, Mathematical problems in classical and non-Newtonian fluid mechanics, Hemodynamical flows, Oberwolfach Semin., vol. 37, Birkhäuser, Basel, 2008, pp. 121-273, DOI 10.1007/978-3-7643-7806-6_3. MR2410706

[58] G. P. Galdi, On the steady self-propelled motion of a body in a viscous incompressible fluid, Arch. Ration. Mech. Anal. 148 (1999), no. 1, 53-88, DOI 10.1007/s002050050156. MR 1715453

[59] G. P. Galdi, On the motion of a rigid body in a viscous liquid: a mathematical analysis with applications, Handbook of mathematical fluid dynamics, Vol. I, North-Holland, Amsterdam, 2002, pp. 653-791. MR 1942470

[60] G. P. Galdi and A. L. Silvestre, Strong solutions to the problem of motion of a rigid body in a Navier-Stokes liquid under the action of prescribed forces and torques, Nonlinear problems in mathematical physics and related topics, I, Int. Math. Ser. (N. Y.), vol. 1, Kluwer/Plenum, New York, 2002, pp. 121-144, DOI 10.1007/978-1-4615-0777-2_8. MR1970608

[61] G. P. Galdi and A. L. Silvestre, The steady motion of a Navier-Stokes liquid around a rigid body, Arch. Ration. Mech. Anal. 184 (2007), no. 3, 371-400, DOI 10.1007/s00205-006-00264. MR2299756

[62] D. Gérard-Varet and M. Hillairet, Computation of the drag force on a sphere close to a wall: the roughness issue, ESAIM Math. Model. Numer. Anal. 46 (2012), no. 5, 1201-1224, DOI 10.1051/m2an/2012001. MR2916378

[63] D. Gérard-Varet, M. Hillairet, and C. Wang, The influence of boundary conditions on the contact problem in a 3D Navier-Stokes flow (English, with English and French summaries), J. Math. Pures Appl. (9) 103 (2015), no. 1, 1-38, DOI 10.1016/j.matpur.2014.03.005. MR 3281946

[64] D. Gérard-Varet and M. Hillairet, Existence of weak solutions up to collision for viscous fluid-solid systems with slip, Comm. Pure Appl. Math. 67 (2014), no. 12, 2022-2075, DOI 10.1002/cpa.21523. MR3272367

[65] O. Glass and F. Sueur, Uniqueness results for weak solutions of two-dimensional fluid-solid systems, Arch. Ration. Mech. Anal. 218 (2015), no. 2, 907-944, DOI 10.1007/s00205-0150876-8. MR 3375542

[66] R. Glowinski, Finite element methods for incompressible viscous flow, Handbook of numerical analysis, Vol. IX, Handb. Numer. Anal., IX, North-Holland, Amsterdam, 2003, pp. 31176. MR2009826

[67] M. Geissert, K. Götze, and M. Hieber, $L^{p}$-theory for strong solutions to fluid-rigid body interaction in Newtonian and generalized Newtonian fluids, Trans. Amer. Math. Soc. 365 (2013), no. 3, 1393-1439, DOI 10.1090/S0002-9947-2012-05652-2. MR3003269

[68] C. Grandmont, M. Lukáčová-Medvid'ová, and Š. Nečasová, Mathematical and numerical analysis of some FSI problems, Fluid-structure interaction and biomedical applications, Adv. Math. Fluid Mech., Birkhäuser/Springer, Basel, 2014, pp. 1-77. MR.3329017

[69] C. Grandmont, Existence for a three-dimensional steady state fluid-structure interaction problem, J. Math. Fluid Mech. 4 (2002), no. 1, 76-94, DOI 10.1007/s00021-002-8536-9. MR.1891075

[70] C. Grandmont, Existence of weak solutions for the unsteady interaction of a viscous fluid with an elastic plate, SIAM J. Math. Anal. 40 (2008), no. 2, 716-737, DOI 10.1137/070699196. MR2438783 
[71] C. Grandmont and M. Hillairet, Existence of global strong solutions to a beam-fluid interaction system, Arch. Ration. Mech. Anal. 220 (2016), no. 3, 1283-1333, DOI 10.1007/s00205015-0954-y. MR3466847

[72] C. Grandmont and Y. Maday, Existence for an unsteady fluid-structure interaction problem, M2AN Math. Model. Numer. Anal. 34 (2000), no. 3, 609-636, DOI 10.1051/m2an:2000159. MR. 1763528

[73] B. E. Griffith, On the volume conservation of the immersed boundary method, Commun. Comput. Phys. 12 (2012), no. 2, 401-432, DOI 10.4208/cicp.120111.300911s. MR2897145

[74] B. E. Griffith, R. D. Hornung, D. M. McQueen, and C. S. Peskin, An adaptive, formally second order accurate version of the immersed boundary method, J. Comput. Phys. 223 (2007), no. 1, 10-49, DOI 10.1016/j.jcp.2006.08.019. MR.2314380

[75] B. E. Griffith, Immersed boundary model of aortic heart valve dynamics with physiological driving and loading conditions, Int. J. Numer. Methods Biomed. Eng. 28 (2012), no. 3, 317-345, DOI 10.1002/cnm.1445. MR2910281

[76] B. E. Griffith, X.-Y. Luo, D. M. McQueen, and C. S. Peskin, Simulating the fluid dynamics of natural and prosthetic heart valves using the immersed boundary method, Internat. J. Appl. Mech. 1 (2009), no. 1, 137-177.

[77] M. Lanzendörfer and J. Hron, On multiple solutions to the steady flow of incompressible fluids subject to do-nothing or constant traction boundary conditions on artificial boundaries, J. Math. Fluid Mech. 22 (2020), no. 1, Paper No. 11, 18, DOI 10.1007/s00021-019-0472-z. MR 4046052

[78] G. Guidoboni, R. Glowinski, N. Cavallini, and S. Canic, Stable loosely-coupled-type algorithm for fluid-structure interaction in blood flow, J. Comput. Phys. 228 (2009), no. 18, 6916-6937, DOI 10.1016/j.jcp.2009.06.007. MR2567876

[79] G. Guidoboni, M. Guidorzi, and M. Padula, Continuous dependence on initial data in fluidstructure motions, J. Math. Fluid Mech. 14 (2012), no. 1, 1-32, DOI 10.1007/s00021-0100031-0. MR2891187

[80] M. D. Gunzburger, H.-C. Lee, and G. A. Seregin, Global existence of weak solutions for viscous incompressible flows around a moving rigid body in three dimensions, J. Math. Fluid Mech. 2 (2000), no. 3, 219-266, DOI 10.1007/PL00000954. MR1781915

[81] M. Hillairet, Lack of collision between solid bodies in a 2D incompressible viscous flow, Comm. Partial Differential Equations 32 (2007), no. 7-9, 1345-1371, DOI 10.1080/03605300601088740. MR2354496

[82] M. Hillairet and T. Takahashi, Collisions in three-dimensional fluid structure interaction problems, SIAM J. Math. Anal. 40 (2009), no. 6, 2451-2477, DOI 10.1137/080716074. MR2481302

[83] K.-H. Hoffmann and V. N. Starovoitov, On a motion of a solid body in a viscous fluid. Two-dimensional case, Adv. Math. Sci. Appl. 9 (1999), no. 2, 633-648. MR1725677

[84] A. Hundertmark-Zaušková, M. Lukáčová-Medviďová, and G. Rusnáková, Fluid-structure interaction for shear-dependent non-Newtonian fluids, Topics in mathematical modeling and analysis, Jindřich Nečas Cent. Math. Model. Lect. Notes, vol. 7, Matfyzpress, Prague, 2012, pp. 109-158. MR2962823

[85] A. Hundertmark-Zaušková, M. Lukáčová-Medviďová, and Š. Nečasová, On the existence of weak solution to the coupled fluid-structure interaction problem for non-Newtonian sheardependent fluid, J. Math. Soc. Japan 68 (2016), no. 1, 193-243, DOI 10.2969/jmsj/06810193. MR.3454559

[86] T. J. R. Hughes, J. A. Cottrell, and Y. Bazilevs, Isogeometric analysis: CAD, finite elements, NURBS, exact geometry and mesh refinement, Comput. Methods Appl. Mech. Engrg. 194 (2005), no. 39-41, 4135-4195, DOI 10.1016/j.cma.2004.10.008. MR.2152382

[87] T. J. R. Hughes, W. K. Liu, and T. K. Zimmermann, Lagrangian-Eulerian finite element formulation for incompressible viscous flows, Comput. Methods Appl. Mech. Engrg. 29 (1981), no. 3, 329-349, DOI 10.1016/0045-7825(81)90049-9. MR659925

[88] M. Ignatova, I. Kukavica, I. Lasiecka, and A. Tuffaha, On well-posedness for a free boundary fluid-structure model, J. Math. Phys. 53 (2012), no. 11, 115624, 13, DOI 10.1063/1.4766724. MR.3026569

[89] M. Ignatova, I. Kukavica, I. Lasiecka, and A. Tuffaha, On well-posedness and small data global existence for an interface damped free boundary fluid-structure model, Nonlinearity 27 (2014), no. 3, 467-499, DOI 10.1088/0951-7715/27/3/467. MR.3168261 
[90] M. Krafczyk, J. Tolke, E. Rank, and M. Schulz. Two-dimensional simulation of fluidstructure interaction using lattice-Boltzmann methods. Computers \& Structures, 79 (2001), 2031-2037.

[91] I. Kukavica and A. Tuffaha, Solutions to a fluid-structure interaction free boundary problem, Discrete Contin. Dyn. Syst. 32 (2012), no. 4, 1355-1389, DOI 10.3934/dcds.2012.32.1355. MR.2851902

[92] I. Kukavica and A. Tuffaha, Well-posedness for the compressible Navier-Stokes-Lamé system with a free interface, Nonlinearity 25 (2012), no. 11, 3111-3137, DOI 10.1088/09517715/25/11/3111. MR2991431

[93] I. Kukavica, A. Tuffaha, and M. Ziane, Strong solutions for a fluid structure interaction system, Adv. Differential Equations 15 (2010), no. 3-4, 231-254. MR2588449

[94] O. A. Ladyzhenskaya. Initial-boundary problem for Navier-Stokes equations in domains with time-varying boundaries, in Boundary Value Problems of Mathematical Physics and Related Aspects of Function Theory, pp. 35-46, Springer, 1970.

[95] D. Lengeler and M. Růžička, Weak solutions for an incompressible Newtonian fluid interacting with a Koiter type shell, Arch. Ration. Mech. Anal. 211 (2014), no. 1, 205-255, DOI 10.1007/s00205-013-0686-9. MR3147436

[96] J. Lequeurre, Existence of strong solutions to a fluid-structure system, SIAM J. Math. Anal. 43 (2011), no. 1, 389-410, DOI 10.1137/10078983X. MR.2765696

[97] D. Maity and M. Tucsnak, $L^{p}-L^{q}$ maximal regularity for some operators associated with linearized incompressible fluid-rigid body problems, Mathematical analysis in fluid mechanicsselected recent results, Contemp. Math., vol. 710, Amer. Math. Soc., Providence, RI, 2018, pp. 175-201, DOI 10.1090/conm/710/14370. MR3818674

[98] J. Málek, J. Nečas, M. Rokyta, and M. Růžička, Weak and measure-valued solutions to evolutionary PDEs, Applied Mathematics and Mathematical Computation, vol. 13, Chapman \& Hall, London, 1996. MR 1409366

[99] A. Mikelić, Rough boundaries and wall laws, Qualitative properties of solutions to partial differential equations, Jindřich Nečas Cent. Math. Model. Lect. Notes, vol. 5, Matfyzpress, Prague, 2009, pp. 103-134. MR 2962831

[100] A. Mikelić, Š. Nečasová, and M. Neuss-Radu, Effective slip law for general viscous flows over an oscillating surface, Math. Methods Appl. Sci. 36 (2013), no. 15, 2086-2100, DOI 10.1002/mma.2923. MR3108828

[101] A. Moussa, Some variants of the classical Aubin-Lions lemma, J. Evol. Equ. 16 (2016), no. 1, 65-93, DOI 10.1007/s00028-015-0293-3. MR.3466213

[102] B. Muha and S. Canić, Existence of a weak solution to a nonlinear fluid-structure interaction problem modeling the flow of an incompressible, viscous fluid in a cylinder with deformable walls, Arch. Ration. Mech. Anal. 207 (2013), no. 3, 919-968, DOI 10.1007/s00205-012-05855. MR3017292

[103] B. Muha and S. Canić, A nonlinear, 3D fluid-structure interaction problem driven by the time-dependent dynamic pressure data: a constructive existence proof, Commun. Inf. Syst. 13 (2013), no. 3, 357-397, DOI 10.4310/CIS.2013.v13.n3.a4. MR3226949

[104] B. Muha and S. Canić, Existence of a solution to a fluid-multi-layered-structure interaction problem, J. Differential Equations 256 (2014), no. 2, 658-706, DOI 10.1016/j.jde.2013.09.016. MR.3121710

[105] B. Muha and S. Canić, Fluid-structure interaction between an incompressible, viscous 3D fluid and an elastic shell with nonlinear Koiter membrane energy, Interfaces Free Bound. 17 (2015), no. 4, 465-495, DOI 10.4171/IFB/350. MR3450736

[106] B. Muha and S. Canić, Existence of a weak solution to a fluid-elastic structure interaction problem with the Navier slip boundary condition, J. Differential Equations 260 (2016), no. 12, 8550-8589, DOI 10.1016/j.jde.2016.02.029. MR3482692

[107] B. Muha and S. Čanić, A generalization of the Aubin-Lions-Simon compactness lemma for problems on moving domains, J. Differential Equations 266 (2019), no. 12, 8370-8418, DOI 10.1016/j.jde.2018.12.030. MR.3944259

[108] B. Muha, Š. Nečasová, and A. Radošević, A uniqueness result for $3 D$ incompressible fluidrigid body interaction problem, arXiv:1904.05102v1 (submitted 2019).

[109] C. Navier. Sur les lois de léquilibre et du mouvement des corps elastiques, Mémoires de l'Académie des Sciences de l'Institut de France, vol. 369, 1827. 
[110] P. Nägele, M. Růžička, and D. Lengeler, Functional setting for unsteady problems in moving domains and applications, Complex Var. Elliptic Equ. 62 (2017), no. 1, 66-97, DOI 10.1080/17476933.2016.1203911. MR3575853

[111] P Nägele. Monotone operator theory for unsteady problems on non-cylindrical domains [dissertation]. Freiburg: Albert-Ludwigs-Universiät Freiburg, 2015.

[112] J. Neustupa and P. Penel, A weak solvability of the Navier-Stokes equation with Navier's boundary condition around a ball striking the wall, Advances in mathematical fluid mechanics, Springer, Berlin, 2010, pp. 385-407, DOI 10.1007/978-3-642-04068-9_24. MR2665044

[113] C. S. Peskin, Numerical analysis of blood flow in the heart, J. Comput. Phys. 25 (1977), no. 3, 220-252, DOI 10.1016/0021-9991(77)90100-0. MR490027

[114] C. S. Peskin and D. M. McQueen, A three-dimensional computational method for blood flow in the heart. I. Immersed elastic fibers in a viscous incompressible fluid, J. Comput. Phys. 81 (1989), no. 2, 372-405, DOI 10.1016/0021-9991(89)90213-1. MR994353

[115] S. Piperno, C. Farhat, and B. Larrouturou, Partitioned procedures for the transient solution of coupled aeroelastic problems. I. Model problem, theory and two-dimensional application, Comput. Methods Appl. Mech. Engrg. 124 (1995), no. 1-2, 79-112, DOI 10.1016/00457825(95)92707-9. MR1342174

[116] J.-P. Raymond and M. Vanninathan, A fluid-structure model coupling the Navier-Stokes equations and the Lamé system (English, with English and French summaries), J. Math. Pures Appl. (9) 102 (2014), no. 3, 546-596, DOI 10.1016/j.matpur.2013.12.004. MR.3249722

[117] A. Quarteroni, M. Tuveri, and A. Veneziani, Computational vascular fluid dynamics: problems, models and methods. Survey article, Comput. Visual. Sci. 2 (2000), 163-197.

[118] A. Quarteroni and A. Valli, Domain decomposition methods for partial differential equations, Numerical Mathematics and Scientific Computation, The Clarendon Press, Oxford University Press, New York, 1999. Oxford Science Publications. MR 1857663

[119] M. Růžička, Multipolar materials, Workshop on the Mathematical Theory of Nonlinear and Inelastic Material Behaviour (Darmstadt, 1992), Bonner Math. Schriften, vol. 239, Univ. Bonn, Bonn, 1993, pp. 53-64. MR1290373

[120] J. O. Sather, The initial bounndary-value problem for the Navier-Stokes equations in regions with moving boundaries, ProQuest LLC, Ann Arbor, MI, 1963. Thesis (Ph.D.)-University of Minnesota. MR2613780

[121] N. Sauer, The steady state Navier-Stokes equations for incompressible flows with rotating boundaries, Proc. Roy. Soc. Edinburgh Sect. A 110 (1988), no. 1-2, 93-99, DOI 10.1017/S0308210500024896. MR963844

[122] J. A. San Martín, V. Starovoitov, and M. Tucsnak, Global weak solutions for the twodimensional motion of several rigid bodies in an incompressible viscous fluid, Arch. Ration. Mech. Anal. 161 (2002), no. 2, 113-147, DOI 10.1007/s002050100172. MR.1870954

[123] J. Serrin, The initial value problem for the Navier-Stokes equations, Nonlinear Problems (Proc. Sympos., Madison, Wis., 1962), Univ. of Wisconsin Press, Madison, Wis., 1963, pp. 69-98. MR0150444

[124] J. Simon, Compact sets in the space $L^{p}(0, T ; B)$, Ann. Mat. Pura Appl. (4) 146 (1987), 65-96, DOI 10.1007/BF01762360. MR916688

[125] V. N. Starovoltov, On the nonuniqueness of the solution of the problem of the motion of a rigid body in a viscous incompressible fluid (Russian, with English and Russian summaries), Zap. Nauchn. Sem. S.-Peterburg. Otdel. Mat. Inst. Steklov. (POMI) 306 (2003), no. Kraev. Zadachi Mat. Fiz. i Smezh. Vopr. Teor. Funktsii. 34, 199-209, 231-232, DOI 10.1007/s10958005-0384-8; English transl., J. Math. Sci. (N.Y.) 130 (2005), no. 4, 4893-4898. MR2065504

[126] T. Takahashi, Analysis of strong solutions for the equations modeling the motion of a rigidfluid system in a bounded domain, Adv. Differential Equations 8 (2003), no. 12, 1499-1532. MR2029294

[127] R. Temam, Navier-Stokes equations: Theory and numerical analysis, Revised edition, Studies in Mathematics and its Applications, vol. 2, North-Holland Publishing Co., AmsterdamNew York, 1979. With an appendix by F. Thomasset. MR603444

[128] C. Wang, Strong solutions for the fluid-solid systems in a 2-D domain, Asymptot. Anal. 89 (2014), no. 3-4, 263-306, DOI 10.3233/asy-141230. MR3266142

[129] Y. Wang, A. Quaini, and S. Čanić, A higher-order discontinuous Galerkin/arbitrary Lagrangian Eulerian partitioned approach to solving fluid-structure interaction problems with 
incompressible, viscous fluids and elastic structures, J. Sci. Comput. 76 (2018), no. 1, 481520, DOI 10.1007/s10915-017-0629-y. MR3812977

[130] H. F. Weinberger, On the steady fall of a body in a Navier-Stokes fluid, Partial differential equations (Proc. Sympos. Pure Math., Vol. XXIII, Univ. California, Berkeley, Calif., 1971), Amer. Math. Soc., Providence, R. I., 1973, pp. 421-439. MR0416234

[131] T. Wick, Variational-Monolithic ALE Fluid-Structure Interaction: Comparison of Computational Cost and Mesh Regularity Using Different Mesh Motion Techniques, in Modeling, Simulation and Optimization of Complex Processes (Bock H., Phu H., Rannacher R., Schlöder J., eds.), HPSC 2015. Springer, Cham.

[132] G. Zhou and N. Saito, The Navier-Stokes equations under a unilateral boundary condition of Signorini's type, J. Math. Fluid Mech. 18 (2016), no. 3, 481-510, DOI 10.1007/s00021016-0248-7. MR3537904

Department of Mathematics, University of California, Berkeley, California

Email address: canics@berkeley.edu 